\title{
The Actin Nucleator Cobl Is Crucial for Purkinje Cell Development and Works in Close Conjunction with the F-Actin Binding Protein Abp1
}

\author{
Natja Haag, ${ }^{1}$ Lukas Schwintzer, ${ }^{1}$ Rashmi Ahuja, ${ }^{1}$ Nicole Koch, ${ }^{1}$ Julia Grimm, ${ }^{1}$ Heike Heuer, ${ }^{2}$ Britta Qualmann, ${ }^{1}$ \\ and Michael M. Kessels ${ }^{1}$ \\ ${ }^{1}$ Institute for Biochemistry I, Jena University Hospital-Friedrich Schiller University Jena, D-07743 Jena, Germany and ${ }^{2}$ Research Group \\ Neuroendocrinology, Leibniz Institute for Age Research/Fritz Lipmann Institute (FLI), 07745 Jena, Germany
}

Cortical actin dynamics shapes cells. To generate actin filaments, cells rely on actin nucleators. Cobl is a novel, brain-enriched, WH2 domain-based actin nucleator, yet, its functions remained largely elusive. Here, we reveal that Cobl plays a crucial role in Purkinje cell development using gene gun transfections within intact murine cerebellar contexts. Cobl deficiency impaired proper dendritic arborization of Purkinje cells and led to low-complexity arbors. Branch point numbers and density and especially higher order branching were strongly affected. Our efforts to reveal how Cobl is physically and functionally integrated into the cortical actin cytoskeleton showed that all Cobl loss-of-function phenotypes were exactly mirrored by knockdown of the F-actin-binding protein Abp1. By subcellular fractionations, protein interaction analyses, subcellular reconstitutions of protein complexes, colocalization studies in cells and tissues, and by functional analyses in neuronal morphogenesis we demonstrate that both proteins associate and work with each other closely. Coblmediated dendritic branch induction in hippocampal neurons critically relied on Abp1. Our study highlights that the functions of Abp1 are distinct from those of the Cobl-binding protein syndapin I. The importance of Cobl/Abpl complex formation and of Abpl-mediated F-actin association was highlighted by functional rescue experiments demonstrating that a Cobl mutant deficient for Abp1 binding and an Abp1 mutant supporting Cobl association but lacking the F-actin binding ability failed to rescue the respective loss-of-function phenotypes. Thus, F-actin-anchored Cobl/Abpl complexes seem crucial for neuromorphogenesis processes, particularly for the postnatal arborization of Purkinje cells representing the source for all motor coordination in the cerebellar cortex.

\section{Introduction}

Shaping the morphology of defined membrane areas and of entire cells is crucial for many developmental processes. The astonishing morphological intricacy neurons acquire during their differentiation is a structural basis for the complex architecture of the neuronal networks, cellular arrays, and layers required for higher brain functions. Purkinje cells are prominent examples for this complexity. These neurons send projections to the deep cerebellar nuclei and constitute the sole output of all motor coordination in the cerebellar cortex. To fulfill their function, Purkinje cells develop one of the most elaborate dendritic arbors among all neurons and thus belong to the largest neurons of the vertebrate brain.

\footnotetext{
Received Feb. 21, 2012; revised Sept. 27, 2012; accepted 0ct. 15, 2012.

Author contributions: B.Q., and M.M.K. designed research; N.H., L.S., R.A., N.K., and J.G. performed research;H.H. contributed unpublished reagents/analytic tools; N.H., L.S., R.A., N.K., and J.G. analyzed data; N.H., L.S., B.Q., and M.M.K. wrote the paper.

This work was supported by grants from the Deutsche Forschungsgemeinschaft to B.Q. (Qu116/6-1), to M.M.K. (KE685/3-1), and to both (Qu116/7-1). We thank Annett Kreusch, Christin Scharf, and Michaela Öhler for their excellent technical assistance as well as Dr. Antje Hübner for her support.

The authors declare no competing financial interests.

Correspondence should be addressed to Michael M. Kessels or Britta Qualmann, Institute for Biochemistry I, Jena University Hospital-Friedrich Schiller University Jena, Nonnenplan 2-4, D-07743 Jena, Germany. E-mail: britta.qualmann@mti.uni-jena.de or michael.kessels@mti.uni-jena.de.

R. Ahuja's present address: Institute of Biotechnology P.0. Box 56, University of Helsinki, 00014 Helsinki, Finland.

DOI:10.1523/JNEUROSCI.0843-12.2012

Copyright $\odot 2012$ the authors $\quad 0270-6474 / 12 / 3217842-15 \$ 15.00 / 0$
}

Morphogenesis of the different types of neurons is steered by a complex interplay of cell-autonomous and extracellular signals (Dotti et al., 1988; Sotelo, 2004; Yuste and Bonhoeffer, 2004). It is thought that one major target of these signals is the cytoskeleton, as coordinated functions of microtubules and actin filaments are capable of creating the mechanical forces required for cell shape remodeling (Georges et al., 2008; Conde and Cáceres, 2009; Poulain and Sobel, 2010; Kessels et al., 2011). Force generation by the actin cytoskeleton either requires motor proteins or locally restricted actin filament formation. The rate-limiting step of de novo actin filament formation is the assembly of actin nuclei, onto which further actin monomers can then add spontaneously. Until today, only a very limited number of actin nucleating machineries are known. These include the Arp $2 / 3$ complex, the formin family, and a novel class of actin nucleators using Wiskott-Aldrich homology 2 (WH2) domains for actin binding and assembly (for reviews, see Pollard, 2007; Qualmann and Kessels, 2009; Campellone and Welch, 2010; Chesarone et al., 2010). The functions of this novel class of actin nucleators are only emerging, as examinations of the endogenous proteins and knowledge of interactions with other cellular components in addition to actin as well as loss-of-function studies addressing the roles of the WH2 domain-based nucleators both at the cellular and at the organ or animal level are still sparse. 
Here we show that the WH2 domain-based actin nucleator Cordon-Bleu (Cobl), which assembles non-bundled, unbranched filaments in in vitro reconstitutions and has the power to shape cells (Ahuja et al., 2007), is highly expressed in Purkinje cells of the cerebellum and plays a crucial role in postnatal Purkinje cell arborization, as unraveled by loss-of-function studies in organotypic slice cultures of developing cerebella. Our analyses strongly suggest that Cobl elicits its functions via interactions with the F-actin binding protein Abp1 (Kessels et al., 2000). Comparative loss-of-function analyses and rescue experiments with mutant Cobl and Abp1 proteins show that the functions of $\mathrm{Cobl} / \mathrm{Abp} 1$ complexes are distinct from Cobl complexes with the membrane curvature-associating F-BAR protein syndapin I (Schwintzer et al., 2011) and require direct Cobl/Abp1 complex formation and Abp1-mediated F-actin association. In line with such intimate cooperation of Cobl and Abp1, developmental defects observed upon Cobl knockdown were phenocopied by Abp1 deficiency. This highlights the importance of Cobl/Abp1 complexes for proper development of cerebellar architecture.

\section{Materials and Methods}

DNA constructs and recombinant proteins. Plasmids encoding for myctagged Abp 1 full-length, GST-Abp $1 \mathrm{SH} 3$ domain, and the corresponding G422R/P425L mutant were described in Kessels et al. (2000, 2001), respectively. Flag-Abp1 full-length was generated by subcloning into a pCMV-Tag2 vector (Stratagene). Mitochondria-targeted GFP-tagged Abp1 SH3 domain was generated by subcloning into a green fluorescent protein (GFP) derivative of the Mito-Flag-vector described previously (Kessels and Qualmann, 2002). Yeast-2-hybrid Abp1 full-length and Abp1 $\Delta$ SH3 (amino acids 1-370) prey constructs were generated by PCR using EcoRI and BamHI sites for cloning into pGADT7 (BD Bioscience). All PCR-generated constructs were sequenced.

Cobl expression constructs used in this study were described previously (Ahuja et al., 2007; Schwintzer et al., 2011) and generated by PCR and by primer annealing, respectively. Cobl sequences were cloned into pCMV-Tag 2 carrying GFP (Ahuja et al., 2007), into a pCMV-based mCherry vector or into pEGFP vectors (Clontech).

RNAi plasmids that are directed against rat and mouse Abp1 and Cobl and coexpress GFP and red fluorescent protein (RFP), respectively, were described previously and characterized for specificity (Ahuja et al., 2007; Pinyol et al., 2007). For this study, Cobl RNAi sequences \#1 and \#2 (Ahuja et al., 2007) and a scrambled RNAi sequence (Pinyol et al., 2007) integrated in pRNAT U6.1 and pRNAT H1.1 (GenScript) were used in parallel.

To construct RNAi vectors coexpressing RNAi-insensitive GFP-Cobl full-length $\left(\right.$ GFP-Cobl $\left.{ }^{*}\right)$ and GFP-Cobl $\Delta 8-28, \Delta 369-402(\Delta$ Abplbinding; Cobl $\left.{ }^{\star \Delta \mathrm{Abp} 1}\right)$, respectively, GFP-Cobl constructs insensitive to RNAi sequence \#1 (Ahuja et al., 2007) were generated and used to replace the GFP reporter via flanking Nhe1 and SmaI sites in pRNAT H1.1 and pRNAT U6.1.

Full-length Abp1 resistant to RNAi sequence \#2 (Pinyol et al., 2007) $\left(\mathrm{Abp}^{*}\right)$ was generated by introducing silent mutations into the codons for K61, V62, Y64, A65, F66, and C67. RNAi constructs (Abp1 RNAi \#2 and scrambled) coexpressing Abp1 and a mutant deficient for F-actin binding (Abp1 $\left.{ }^{\Delta \text { Actin }}\right)$ were generated by replacing the GFP of the pRNAT H1.1 vector by GFP-Abp1 full-length and GFP-Abp1 ${ }^{\Delta \text { Actin }}(\Delta \mathrm{aa} 1-281)$, respectively, using the NheI and EcoRI sites of pRNAT H1.1 flanking the GFP.

Plasmids encoding for His-thioredoxin (HisTRX)-tagged Cobl fusion proteins used as antigens for immunizations were constructed by PCR and cloning into pET32 vectors (Novagen). HisTRX-tagged fusion proteins were expressed in Escherichia coli BL21 cells and purified according to Schwintzer et al. (2011). Glutathione $S$-transferase (GST) and GSTfusion proteins were expressed and purified as described previously (Qualmann et al., 1999; Kessels et al., 2000).

Antibodies. Rabbit and guinea pig anti-Abpl and anti-GST antibodies were raised and affinity-purified as described previously (Kessels et al.,
2000; Fenster et al., 2003). Anti-Cobl antibodies were raised against a HisTRX-tagged antigen spanning the amino acid sequences 175-324 $\Delta 262-286$ (ARA) and 750-1005 (DBY) in rabbits and guinea pigs, respectively. Sera were affinity-purified against GST-tagged fusion proteins resolved on preparative gels by SDS-PAGE and immobilized on nitrocellulose membranes according to procedures described previously (Qualmann et al., 1999).

Additionally, anti-HisTRX antibodies were affinity-purified from the same sera using immobilized HisTRX protein. All sera and all affinity-purified antibodies were tested in Western blot analyses with different amounts of recombinant antigen, rat brain cytosol, and GFP-Cobl-expressing HEK293 cell lysates, respectively. Additionally, the antibodies were characterized by immunofluorescence studies of COS-7 cells expressing different GFP-tagged Cobl fusion proteins containing and lacking the antigenic protein sequence, respectively. For characterization of the rabbit anti-Cobl antibodies see Schwintzer et al. (2011). Guinea pig antibodies against Cobl are characterized in this study.

Rabbit anti-Calbindin antibodies (D28K) were from Cell Signaling Technology. Mouse anti-GFAP (G-A-5), anti-Flag (M2), and anti-MAP2 antibodies were from Sigma. Rabbit anti-Flag antibodies were from Zymed. Rabbit anti-GFP (290) antibodies were from Abcam. Monoclonal anti-GFP (B34) and anti-myc (9E10) antibodies were purchased from Babco.

Secondary antibodies used in this study included Alexa Fluor 350, 488, and 568 goat anti-rabbit antibodies; Alexa Fluor 488 and 568 goat antiguinea pig antibodies; Alexa Fluor 350, 488, 568, and 647 goat antimouse antibodies (Invitrogen); and goat anti-rabbit and donkey anti-mouse Cy5 antibodies as well as donkey anti-rabbit- and antiguinea pig-peroxidase, goat anti-mouse-peroxidase (Dianova), DyLight 800-conjugated goat anti-rabbit antibodies (Pierce), and donkey antiguinea pig antibodies coupled to IRDye680 and IRDye800CW, respectively (LI-COR Bioscience).

Coprecipitation analyses. For coprecipitation experiments, GFP-Cobl and Flag-GFP-Cobl fusion proteins were expressed in HEK293 cells, extracted, and incubated with $30 \mu \mathrm{g}$ of purified GST-fusion proteins immobilized on glutathione Sepharose beads, as described previously (Qualmann et al., 1999). Bound proteins were eluted with $20 \mathrm{~mm}$ reduced glutathione, $120 \mathrm{~mm} \mathrm{NaCl}, 50 \mathrm{~mm}$ Tris/HCl pH 8.0, separated by SDSPAGE and analyzed by anti-GFP immunoblotting.

For coprecipitation of endogenous Cobl from cerebellum, P10 mouse cerebella were dissected and homogenized in lysis buffer (10 mM HEPES, pH 7.5, 1 mm EGTA, 0.1 mm $\mathrm{MgCl}_{2}, 1 \%$ (v/v) Triton X-100) containing $100 \mathrm{~mm} \mathrm{NaCl}$ and protease inhibitor Complete EDTA-free (Roche) (1:3 $(\mathrm{w} / \mathrm{v})$ ) using an Ultra Turrax homogenizer (Ika Ultra Turrax T5Fu; $20,000 \mathrm{rpm}, 10 \mathrm{~s}$ ). The homogenates were spun at $100,000 \times \mathrm{g}$ for $1 \mathrm{~h}$. Supernatants were used to precipitate endogenous protein complexes as described above.

All affinity purifications were accompanied by the corresponding control experiments with merely GST attached to the beads.

Coimmunoprecipitations. Heterologous coimmunoprecipitations in the presence of $150 \mathrm{~mm} \mathrm{NaCl}$ were performed with $1.5 \mu \mathrm{g}$ of monoclonal anti-Flag antibody (M2) and $2 \mu \mathrm{g}$ of non-immune mouse IgG (Santa Cruz Biotechnology) immobilized on protein G Sepharose (Santa Cruz Biotechnology) according to Schwintzer et al. (2011). Immunoprecipitates were analyzed by immunoblotting with anti-Flag and anti-GFP antibodies to detect immunoprecipitated and coimmunoprecipitated proteins, respectively.

Lysates for coimmunoprecipitation of endogenous proteins were prepared from brains of 8-week-old male rats homogenized in lysis buffer with $30 \mathrm{~mm} \mathrm{NaCl}$ and protease inhibitor Complete EDTA-free (Roche) $(1: 3(\mathrm{w} / \mathrm{v}))$ using an Ultra Turrax homogenizer. The homogenates were spun at $150,000 \times g$ for $45 \mathrm{~min}$. Supernatants (rat brain extracts; $1 \mathrm{mg}$ of protein was used for each incubation) were used to coimmunoprecipitate endogenous protein complexes with guinea pig anti-Cobl antibodies immobilized on protein A agarose. Immunoprecipitates were washed with lysis buffer supplemented with $30 \mathrm{~mm} \mathrm{NaCl}$, eluted with $4 \times$ SDS sample buffer and analyzed by immunoblotting with rabbit anti-Cobl and rabbit anti-Abp1 antibodies. 
Fractionation of brain extracts. For subcellular fractionation and floatation assays, mouse cerebrum was homogenized in ice-cold lysis buffer with $150 \mathrm{~mm} \mathrm{NaCl}$ and protease inhibitor $(1: 3(\mathrm{w} / \mathrm{v}))$ using a Potter S homogenizer (Sartorius). Lysate (525 $\mu \mathrm{l})$ was supplemented with $175 \mu \mathrm{l}$ of $1 \mathrm{M}$ sucrose in PBS and centrifuged at $1000 \times g\left(3 \mathrm{~min}\right.$ at $\left.4^{\circ} \mathrm{C}\right)$. Two hundred microliters of the resulting supernatant were diluted to $1 \mathrm{ml}$ by adding sucrose $(68.5 \%$ final), placed under two cushions of $65 \%(7 \mathrm{ml})$ and $10 \%$ sucrose $(10 \mathrm{ml})$, and centrifuged overnight at $4^{\circ} \mathrm{C}$ at $143,000 \times$ g. One milliliter fractions were collected from top to bottom. Proteins were precipitated by trichloroacetic acid $(10 \%(\mathrm{v} / \mathrm{v})$ final), washed with cold $80 \%$ ethanol, and solubilized in SDS sample buffer. The lower 12 samples were analyzed by immunoblotting.

Reverse transcription PCR. For RNA isolation, tissue samples from whole mouse brain and cerebellum, respectively, were dissected, snap-frozen, and homogenized by grinding in liquid nitrogen and subsequently resuspended in Trizol reagent (Invitrogen). Two micrograms of DNasetreated (RNase-free DNase kit, Qiagen) total RNA were reverse transcribed using oligo(dT)-primers and RevertAid H Minus Reverse Transcriptase (Fermentas). To test for the absence of contaminating genomic DNA, controls omitting reverse transcriptase were run in parallel. Cobl expression was analyzed by PCR using the exon-spanning primers GGCTCCTGAGAAATCTGTACG ( $\mathrm{fwd}$ ) and CTAAACATTTCT CTTCTGTTGTCC (rev).

Generation and immunolabeling of mouse brain sections. Mice were anesthetized by intraperitoneal injections of a mixture of $72 \mu$ l ketamine hydrochloride ( $50 \mathrm{mg} / \mathrm{ml}), 25.5 \mu \mathrm{l}$ Rompun ( $2 \%$ solution), and $202.5 \mu \mathrm{l}$ $0.9 \%(\mathrm{w} / \mathrm{v}) \mathrm{NaCl}$ and transcardially perfused with $\mathrm{PBS}$ and subsequently with $4 \%$ paraformaldehyde (PFA) in PBS.

Brains were removed, postfixed in $4 \%$ PFA for $16 \mathrm{~h}$ at $4^{\circ} \mathrm{C}$, rinsed with PBS and tap water, and then placed in $30 \%(\mathrm{w} / \mathrm{v})$ sucrose in PBS at $4^{\circ} \mathrm{C}$ until saturation. Brains were sliced sagittally on a sliding microtome (SM 2000R; Leica). The obtained slices $(55 \mu \mathrm{m})$ were collected in $\mathrm{PB}(77.4 \mathrm{~mm}$ $\left.\mathrm{Na}_{2} \mathrm{HPO}_{4}, 22.6 \mathrm{~mm} \mathrm{NaH} \mathrm{PO}_{4}\right)$, swayed in $\mathrm{PB}(2 \times 5 \mathrm{~min})$, and then permeabilized and blocked by incubation with $5 \%(\mathrm{v} / \mathrm{v})$ goat serum, $0.25 \%(\mathrm{v} / \mathrm{v})$ Triton X-100 in PB (block solution) for $2 \mathrm{~h}$ at room temperature (RT). Primary antibody labeling was done in block solution for $48 \mathrm{~h}$ at $4^{\circ} \mathrm{C}$. The slices were then washed and incubated with secondary antibody conjugates for $16 \mathrm{~h}$ at $4^{\circ} \mathrm{C}$. DAPI (30 min, 1:1000 in PB) was used to label nuclei. Finally, slices were transferred into PBS and mounted with Fluoromount-G (Southern Biotech) onto HistoBond slides.

Organotypic culture of cerebellar slices. P9 and P10 mouse pups (NMRI), respectively, were decapitated, brains were quickly dissected out, and cerebella were isolated. Parasagittal cerebellar slices $(250 \mu \mathrm{m}$ thick) were cut on a Mcllwain tissue chopper, subsequently rinsed with CMF-PBS (137 mm NaCl, $4 \mathrm{~mm} \mathrm{KCl}, 11 \mathrm{~mm}$ glucose, $3.6 \mathrm{~mm} \mathrm{NaH} \mathrm{PO}_{4}$, $1.8 \mathrm{mM} \mathrm{KH}_{2} \mathrm{PO}_{4}, 2 \%(\mathrm{v} / \mathrm{v}) \mathrm{NaHCO}_{3}, 0.5 \%$ (v/v) phenol red) and teased apart using fine forceps. After repeated rinsing in CMF-PBS, slices from the medial/vermis part of the cerebellum were transferred onto membranes (Millicell cell culture inserts, $0.4 \mu \mathrm{m}$ pore size, Millipore) and cultured $\left(37^{\circ} \mathrm{C}, 5 \% \mathrm{CO}_{2}\right)$ in six-well plates with Neurobasal medium (Invitrogen) supplemented with $2 \mathrm{~mm}$ GlutaMax (Invitrogen), $1 \times$ B27 serum-free supplement (Invitrogen) and antibiotics (penicillin-streptomycin; Invitrogen) for $\sim 4 \mathrm{~h}$ before transfection.

Biolistic transfection. Transfection of single Purkinje cells in cerebellar tissue sections was performed using the Helios Gene Gun system (BioRad) delivering DNA-coated gold microbeads. Briefly, $1 \mu \mathrm{m}$ gold beads (Bio-Rad) were coated with $50 \mathrm{~mm}$ spermidine followed by precipitation of RNAi plasmids onto the gold beads using $1 \mathrm{M} \mathrm{CaCl}_{2}$. DNA-coated beads were washed several times in 100\% ethanol, coated along the inside wall of Teflon tubing, which was cut into small cartridges. Biolistic bombardments of cerebella were performed using helium pressure pulses (140-160 mmHg). After gene gun transfection, further development of the cerebellar slices in culture was allowed for $42 \mathrm{~h}$.

Immunohistochemistry of organotypic cerebellar slice cultures. Cerebellar slices were fixed in $4 \%$ PFA in PBS for $1.5 \mathrm{~h}$ at RT. The signals of RNAi reporter genes were enhanced by incubation with anti-GFP antibodies. For this purpose, slices were rinsed in PBS and blocked in 10\% normal goat serum, $0.2 \%$ Triton X-100 in PBS for at least $30 \mathrm{~min}$, and then incubated with primary antibodies overnight at $4^{\circ} \mathrm{C}$. After washing in
PBS, secondary antibodies were applied in 1\% normal goat serum, $0.2 \%$ Triton X-100 in PBS for $2 \mathrm{~h}$ at RT. After washing cerebellar slices were mounted in Fluoromount-G

Immunofluorescence microscopy. Primary hippocampal cultures and COS-7 cells were prepared and processed for immunofluorescence microscopy as described previously (Qualmann et al., 2004).

Confocal images were recorded using a Leica TCS SP5 and a Leica TCS NT laser confocal microscope, respectively, or a Zeiss Observer microscope equipped with an ApoTome. Further imaging was done using a Zeiss Axio-Imager.D1 as well as a Zeiss AxioObserver.Z1 microscope both equipped with Zeiss Plan-Apochromat $100 \times / 1.4,63 \times / 1.4,40 \times /$ 1.3 and $20 \times / 0.5$ objectives, respectively. The microscopes were connected to a CCD camera 2.1.1 (Diagnostic Instruments) and to an AxioCam MRm CCD camera (Zeiss), respectively.

Digital images were recorded by AxioVision and SpotSoftware, respectively, and processed by ImageJ and/or Adobe Photoshop.

Morphometric analyses. Primary hippocampal neurons were transfected at 4 days in vitro (DIV 4) with Lipofectamine 2000 (Invitrogen) and fixed $48 \mathrm{~h}$ later. Morphometric measurements were done according to Dharmalingam et al. (2009) and Pinyol et al. (2007). Transfections were repeated several times with independent neuronal preparations. Four experiments with at least 12 GFP- and GFP-Cobl expressing neurons, respectively, were analyzed morphometrically. Transfected cells were not selected but imaged in systematic sweeps across the coverslips. Neurons were identified by anti-MAP2 staining. Then $88-159$ cells for each condition were examined for their number of dendrites and dendritic branch points based on anti-MAP2 immunosignals $(=$ dendrite morphology tracer). A length of $10 \mu \mathrm{m}$ was used as threshold for dendrite and dendritic branch consideration. All data were normalized to the corresponding controls run in parallel in each individual experiment and neuronal preparation. Statistical analysis was performed using ANOVA with Tukey's HSD post test.

For morphometric analyses of Purkinje cells in organotypic cerebellar slice cultures, the following parameters were analyzed: (1) the maximum extension of the dendritic arbor (center of the cell soma to the tip of the longest dendrite), (2) the length of the primary dendrite (border of the cell soma to the first branching point), (3) the area of the molecular layer spanned by the dendritic arbor, (4) the branch points of the dendritic tree, and (5) the orders of dendritic sections within the dendritic arbor. Additionally, the branch point density was calculated. Dendrite order analysis was conducted as follows: starting with the primary dendrite as the first-order dendrite, every dendrite branching off a first-order dendrite was defined as second-order dendrite, and so on. The number of dendrites was plotted as a function of their branching order. Statistical analysis was performed using ANOVA with Tukey's HSD post-test.

\section{Results}

\section{Cobl is highly expressed in Purkinje cells of the cerebellum}

Cobl is a powerful actin nucleator and modulations of Cobl levels were shown to affect the shape of dissociated hippocampal neurons (Ahuja et al., 2007), yet, the functions of Cobl in the context of the developing brain remained unclear. Immunohistochemical analyses of adult mouse brains showed that anti-Cobl immunoreactivity can be found in many areas of the brain but is predominant in the cerebral cortex and the cerebellum (Fig. 1A).

In the cerebellum, both the Purkinje cell layer and the molecular layer were anti-Cobl immunopositive, whereas immunolabeling of the granule cell layer was sparse (Fig. 1B,C). Different control incubations including controls with unrelated antibodies affinity-purified from the same antisera as the anti-Cobl antibodies were imaged in parallel and clearly demonstrated that the anti-Cobl immunolabeling in the cerebellum was specific (Fig. 1D).

Analyses of cerebella during postnatal stages P6, P10, P13, and adulthood showed that the Purkinje cell layer was anti-Cobl immunopositive at all stages (Fig. 1E). In the molecular layer, migrating granule cells were not stained by anti-Cobl antibodies. 

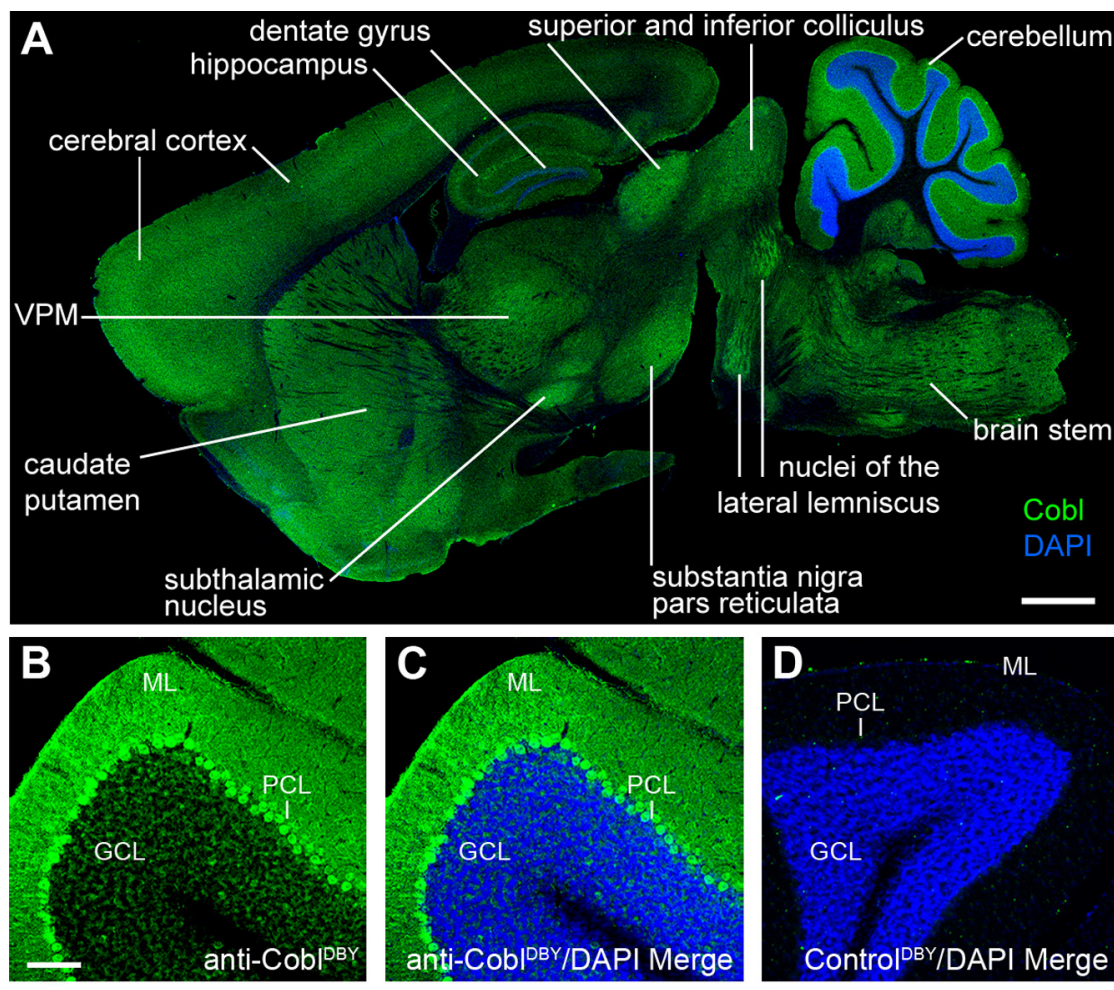

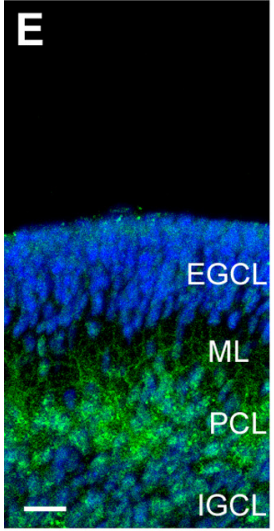

P6

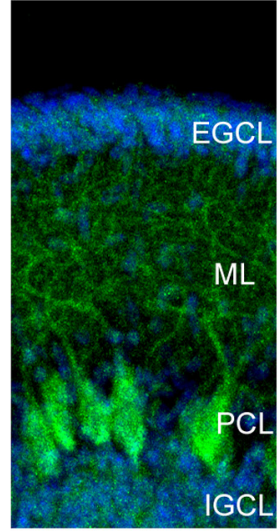

P10

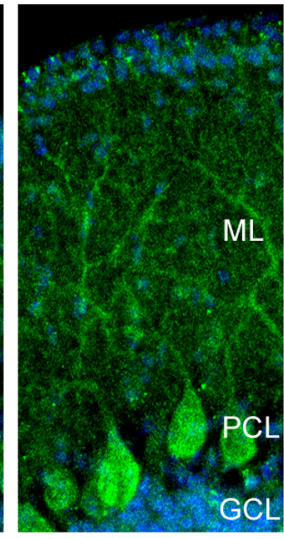

P13

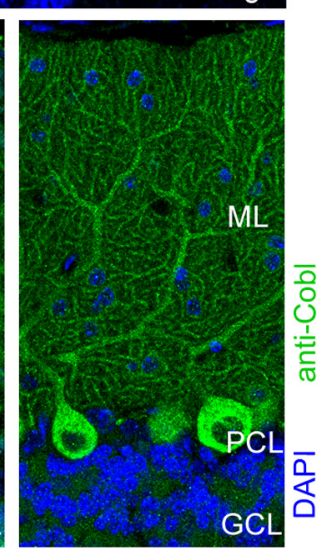

adult

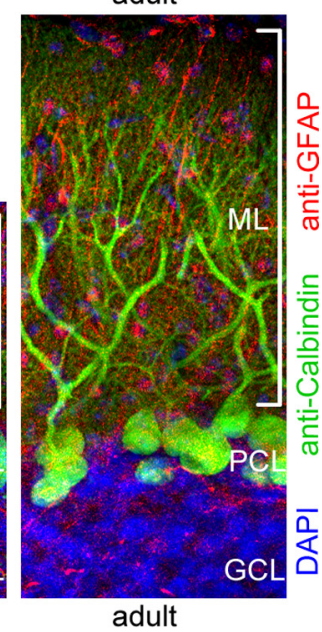

adult
F Whole brain Cerebellum

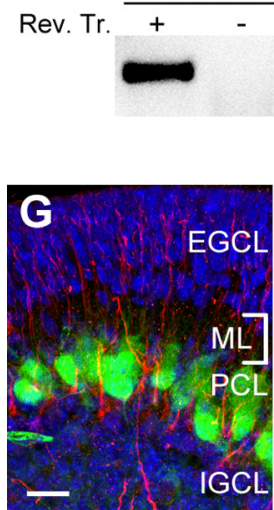

P6

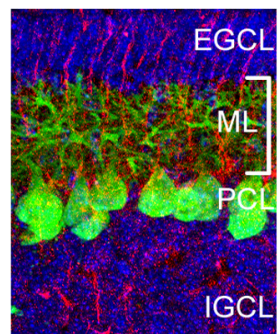

P10

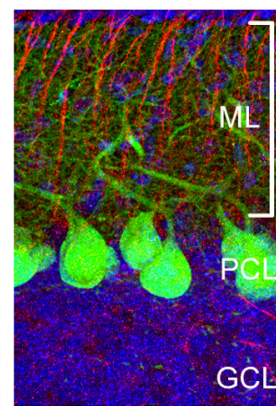

P13
Figure 1. Cobl is highly expressed in the cerebellum. $A$, Anti-Cobl immunolabeling (green) of a sagittal section of an adult mouse brain analyzed by confocal microscopy (composite image). Nuclei are marked with DAPI (blue). VPM, ventral posteromedial nucleus of the thalamus. B, C, Parasagittal slices of adult murine cerebella with anti-Cobl immunoreactivity in Purkinje cells, i.e., in the Purkinje cell layer $(\mathrm{PCL})$ and in the molecular layer $(\mathrm{ML})$. $\boldsymbol{D}$, Incubations with unrelated antibodies affinity-purified from the same serum as anti-Cobl antibodies processed in parallel to the anti-Cobl immunolabelings shown in $\boldsymbol{B}$ and $\boldsymbol{C}$. The lacking
Instead, immunoreactivity in the molecular layer appeared dendritic in nature and thus increased during postnatal development of the cerebellum (Fig. 1E).

Immunodetections of proteins in the cerebellum are often hampered by unspecific stainings, especially of the Purkinje cell and/or the molecular layer. As an antibody-independent proof, reverse transcription-PCR analyses were thus additionally used to confirm the prominent Cobl expression in the cerebellum at the mRNA level (Fig. $1 F$ ).

The anti-Cobl immunosignal in the Purkinje cell layer and in the molecular layer suggested an expression of Cobl in Purkinje cells. Comparative analyses indeed demonstrated that the anti-Cobl immunostaining in the cerebellum reflected an expression in Purkinje cells, as the Purkinje cell marker Calbindin yielded a very similar pattern. Anti-Calbindin immunolabeling also highlighted the Purkinje cell bodies in the Purkinje cell layer and their dendritic trees in the molecular layer. In contrast, anti-GFAP-labeled Bergmann glia tracks in the expanding molecular layer showed a completely different, more linear pattern (Fig. $1 E, G$ ).

Cobl is crucial for proper development of cerebellar Purkinje cells

For proper development of cerebellar circuits, Purkinje cells need to develop an extended, yet almost two-dimensional dendritic tree ensuring contact with the parallel fibers in the molecular layer. This maturation occurs postnatally and strongly expands the molecular layer, which at P6 only has an extension of $\sim 20 \mu \mathrm{m}$, whereas, in the mature mouse brain, the molecular layer is $\sim 10$ times as wide (Fig. 1).

To address the role of the actin nucleator Cobl in Purkinje cell development, we prepared and cultured 250-

immunosignal demonstrates the specificity of the anti-Cob immunostainings in $\boldsymbol{A}-\boldsymbol{C}$. The granule cell layer $(\mathrm{GCL})$ is highlighted by DAPI staining (blue in merged images in ( and $\boldsymbol{D}$ ). $\boldsymbol{E}$, Cobl expression (green) during postnatal stages P6, P10, and P13 and adulthood in the cerebellum. $\boldsymbol{F}$, Reverse transcription$P C R$ confirms the presence of Cobl mRNA in total RNA of mouse brain and cerebellum. Rev. Tr., reverse transcriptase. G, Immunolabeling of Bergmann glia (anti-GFAP, red) and Purkinje cells (anti-Calbindin, green) in parasagittal sections at different time points during the postnatal development of the cerebellum. Blue, DAPI. EGCL, external granule cell layer; IGCL, internal granule cell layer. Note that the anti-Cobl immunosignal in the $P C L$ and in the $M L(E)$ resembles that of the Purkinje cell marker Calbindin (G). Scale bars: $\boldsymbol{A}, 1 \mathrm{~mm} ; \boldsymbol{B}-\boldsymbol{D}, 100 \mu \mathrm{m}$; E, G, $20 \mu \mathrm{m}$ 

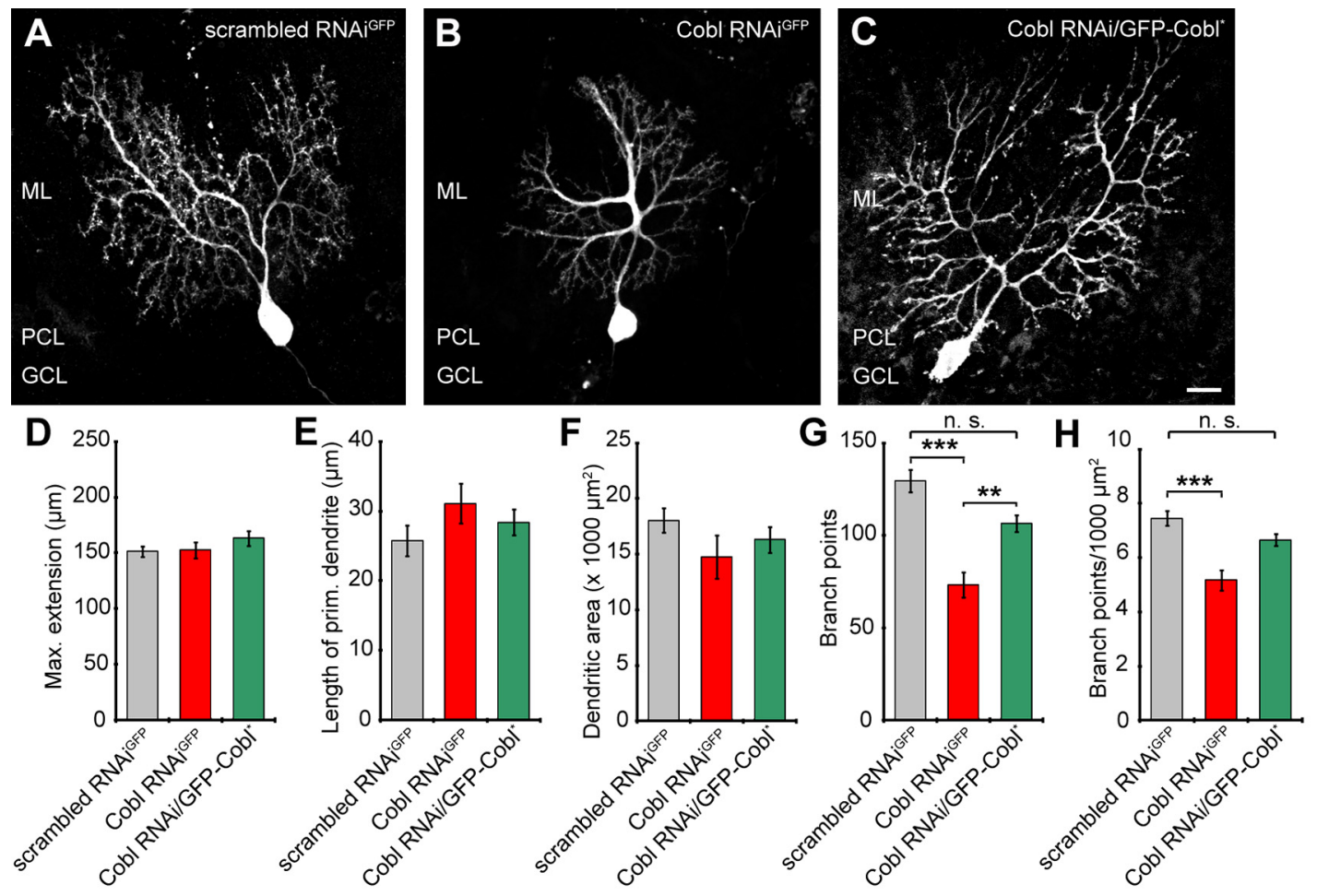

P10
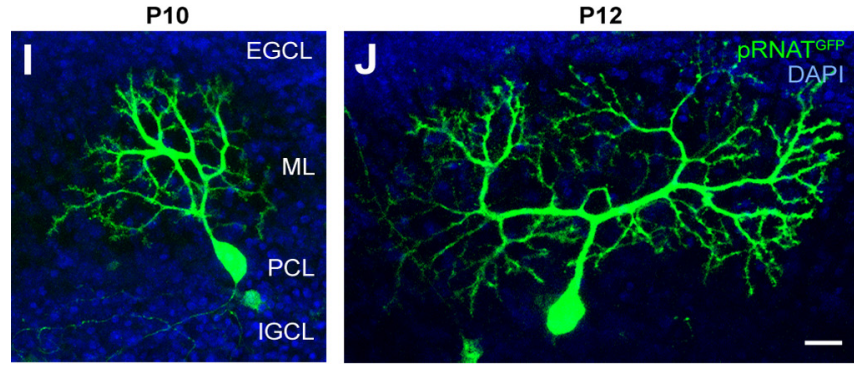

K

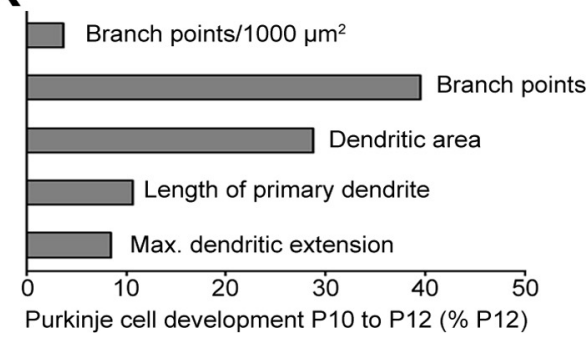

Figure 2. Cobl is required for proper formation of the dendritic Purkinje cell arbor. $A-C$, Parasagittal cerebellar slices $(250 \mu \mathrm{m})$ at P12 showing individual Purkinje cells transfected with plasmids encoding for scrambled RNAi ${ }^{G F P}(\boldsymbol{A})$, Cobl RNAi ${ }^{\text {GFP }}(\boldsymbol{B})$, and Cobl RNAi/GFP-Cobl ${ }^{*}(\boldsymbol{C})$, respectively, $42 \mathrm{~h}$ before fixation. Labeling of cerebellar layers is as in Figure 1. $\boldsymbol{D}-\boldsymbol{H}$, Quantitative evaluation of Purkinje cells in organotypic cerebellar sections. Shown are the maximal extension of the dendritic arbor $(\boldsymbol{D})$, the primary dendrite length $(\boldsymbol{E})$, the area covered by the dendritic arbor $(\boldsymbol{F})$, the number of dendritic branch points $(\boldsymbol{G})$, and the branch point density $(\boldsymbol{H})$. Scrambled RNAi ${ }^{\text {GFP }}, n=34 ; \mathrm{Cobl} \mathrm{RNAi}^{\mathrm{GFP}}, n=21$; Cobl RNAi/GFP-Cobl ${ }^{*}, n=10$. Data are mean \pm SEM. ${ }^{* *} p<0.01,{ }^{* * *} p<0.001$. Note that morphometric data obtained from Cobl RNAi/GFP-Cobl* cells were not significantly different from control cells and that also for the branch point density, statistical significance between Cobl RNAi and the Cobl RNAi/GFP-Cobl* data exists $\left(p=0.0009\right.$; i.e., $\left.{ }^{* * *}\right)$, if only these two values are compared and thus Student's $t$ test is used. $I-\boldsymbol{K}$, Purkinje cell morphology at P10 ( $\left.\boldsymbol{I}\right)$ and P12 $(\mathcal{J})$, as visualized by gene gun transfection of GFP reporters (PRNAT $\left.{ }^{\text {GFP }}\right) 24 \mathrm{~h}$ before fixation and quantitative evaluation of the P10 $-\mathrm{P} 12$ development $(\mathrm{P} 10, n=11 ; \mathrm{P} 12, n=6)(\boldsymbol{K})$. DAPI is shown in blue. Scale bars: $20 \mu \mathrm{m}$.

$\mu$ m-thick parasagittal slices of the cerebellum from mice of different age and analyzed the development of individual Purkinje cells by firing plasmid-coated gold bullets into the tissue (Fig. 2). P10 preparations turned out to be most useful. Quantitative analyses 2 days after biolistic transfection showed that interslice variances of Purkinje cell morphology were moderate and permitted quantitative evaluation of developmental processes using different fluorescent proteins. To address whether Cobl may contribute to cerebellar differentiation, we used established, specific RNAi sequences (Ahuja et al., 2007). Since RNAi was driven by the pRNAT H1.1 vector coexpressing GFP, GFP fluorescence could be used for comparison of the morphologies of single, Cobl-depleted Purkinje cells within otherwise wild-type cerebella.

Cobl-deficient cells showed significantly less elaborate dendritic arbors compared with scrambled RNAi-transfected control cells and also compared with Cobl RNAi cells coexpressing RNAi-insensitive Cobl (GFP-Cobl ${ }^{\star}$ ) (Fig. 2A-C).
Subsequent detailed quantitative analyses of Cobl-deficient Purkinje cells showed that the maximal dendrite extension and the length of the primary dendrites were not affected (Fig. $2 D, E)$. Compared with the scrambled RNAi control, the overall area spanned within the molecular layer also was not significantly reduced (Fig. $2 F$ ). Strikingly, Cobl deficiency led to a strong reduction of the absolute numbers of dendritic branch points when compared with the scrambled RNAi control $\left(-56.9 \% ; p<10^{-6}\right.$; Fig. $\left.2 G\right)$. Since the area of the molecular layer spanned by Cobl-deficient Purkinje cells showed a trend toward reduction when compared with the scrambled RNAi control ( $p=0.118$; Fig. $2 F$ ), we excluded the possibility that the reduction of absolute branch point numbers (Fig. $2 G$ ) was simply due to a smaller dendritic tree. A significant reduction of the branch point density in Cobl-deficient cells $\left(-29.2 \% ; p=0.97 \times 10^{-5}\right.$; Fig. $\left.2 H\right)$ clearly demonstrated that Cobl loss-of-function indeed caused a loss of complexity of the Purkinje cell arbor. 
A
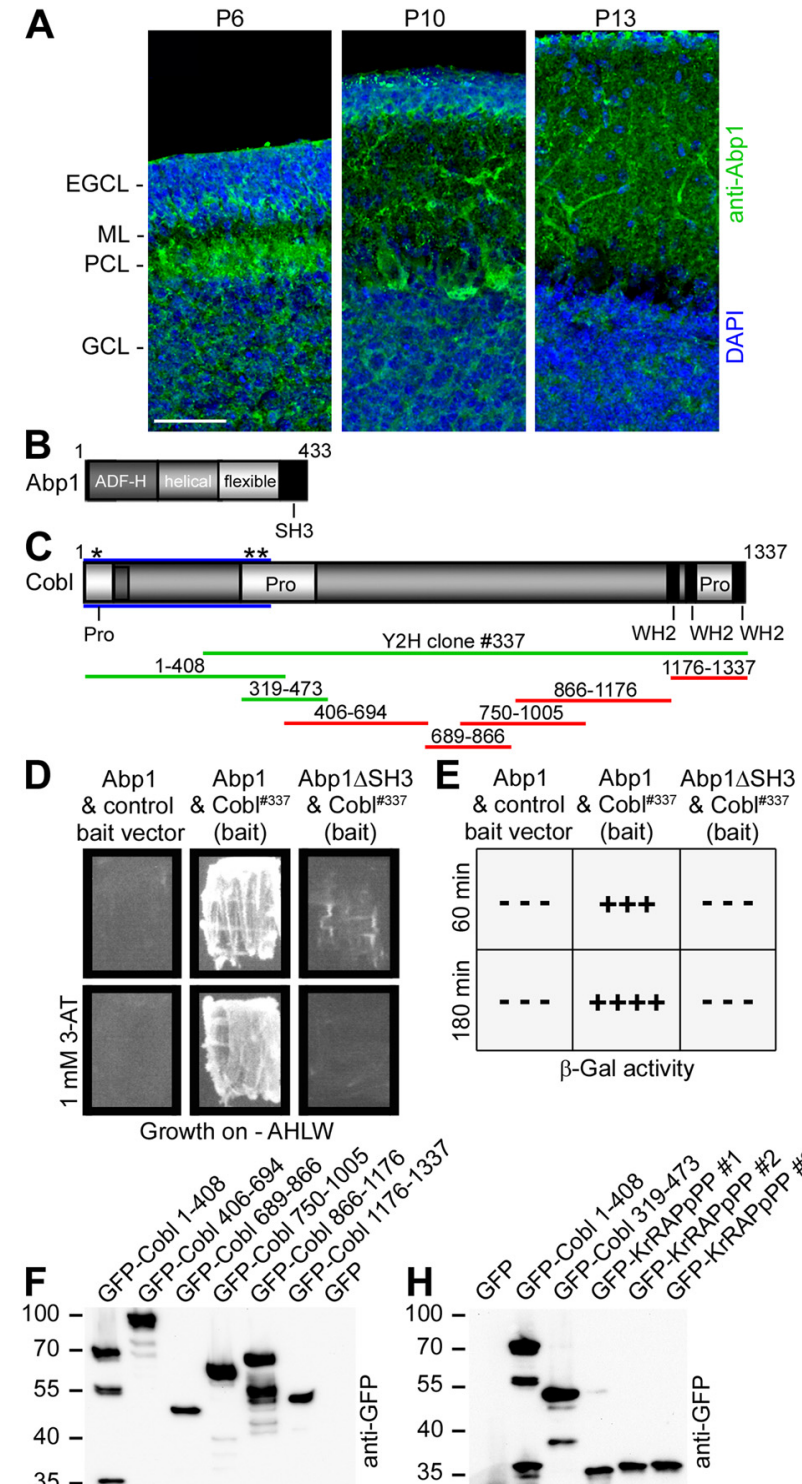

Abp1 Abp1 Abp1 1 SH

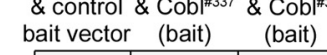
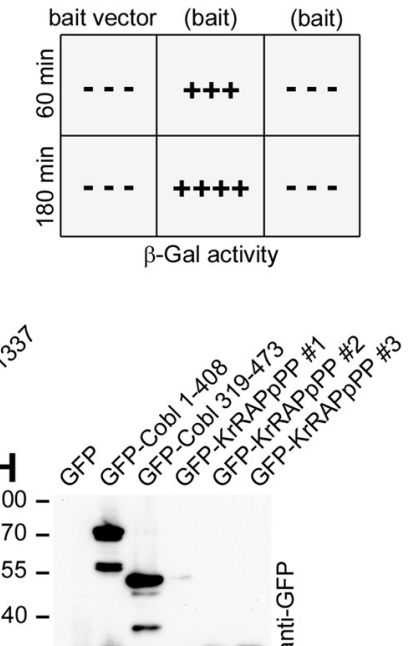
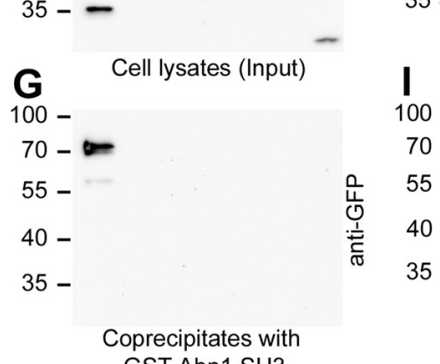

Cell lysates (Input)

$100-$

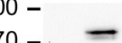

$55-$

$40-$

$35-$

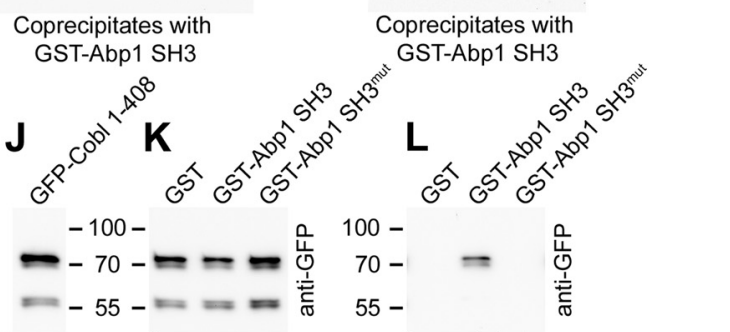

Lysate Supernatants

Coprecipitates

Figure 3. The expression of Abp1 during cerebellum development resembles that of Cobl, and Abp1 associates with the $\mathrm{N}$-terminal region of $\mathrm{Cobl}$ via its $\mathrm{SH} 3$ domain in vitro. $\boldsymbol{A}$, Parasagittal slices of the cerebellum at stages P6, P10, and P13 immunolabeled with anti-Abp1 antibodies (green). Labeling of cerebellar layers as in Figure 1. Blue, DAPI. Scale bar, $50 \mu \mathrm{m}$. $\boldsymbol{B}$, Schematic representation of Abp 1 and its domains. ADF-H, actin-depolymerizing factor homology domain; SH3, Src homology 3 domain. C, Schematic representation of Cobl, its domains

Importantly, the defects of Purkinje cell development observed upon Cobl RNAi were abolished when Cobl expression was restored by coexpression of an RNAi-resistant full-length Cobl, which, however, was unchanged at the amino acid level. Note that when Cobl RNAi and the rescue data are directly compared, statistical significance between Cobl RNAi and the rescue data also exists for the branch point density ( $p=0.009$; i.e., ${ }^{* * *}$; Student's $t$ test). For both phenotype parameters, morphometric data obtained from Cobl RNAi/GFP-Cobl ${ }^{\star}$ cells were not significantly different from control cells (Fig. $2 D-H$ ). The finding that re-expression of Cobl rescues Cobl RNAi phenotypes shows that the RNAi phenotypes are specific.

To analyze the relative impact of Cobl deficiency on Purkinje cell development, we next examined the development of Purkinje cells during the time frame from P10 to P12 used for analyses (Fig. $2 I-K$ ). During this time window, branch point numbers and dendritic area strongly increased, whereas other parameters, such as maximal extension and primary dendrite length, did not change much (Fig. $2 \mathrm{~K}$ ). A comparison of the P10 data to our quantitative analyses (Fig. $2 D-H$ ) revealed that the Cobl RNAiinduced impairments corresponded to a very severe suppression of Purkinje cell arborization during the P10 to P12 time frame. Thus, Cobl is a crucial factor for proper postnatal development of Purkinje cells.

\section{Abp1 is expressed in Purkinje cells and physically interacts with Cobl in vitro}

Recent studies revealed that proper development of the axon of dissociated hippocampal neurons is mediated by Abp1 (Pinyol et al., 2007; Dharmalingam et al., 2009). This actin-binding protein was also suggested to associate with Cobl (Ahuja et al., 2007). Analyses of anti-Abp1 immunostainings of P6, P10, and P13 cerebella showed that Abp1 is highly expressed during all stages of development (Fig. 3A). Importantly, the expression of Abp1 in the cerebellum is predominantly found in the Purkinje cell bodies in the Purkinje cell layer, and in the Purkinje cell dendrites spanning the molecular layer. Although less restricted to these layers, Abp1 immunoreactivity thereby resembles that of Cobl. Interestingly, upon maturation, the localization of Abp1 shifts toward the dendritic arbor of Purkinje cells in the molecular layer and the immunostaining in the cell bodies of Purkinje cells is reduced correspondingly (Fig. 3A). This suggested that Abp1 may play some role in postnatal Purkinje cell arborization.

Yeast-2-hybrid analyses showed that Cobl physically interacts with Abp1. The combination of yeast-2-hybrid clone \#337 (Fig.

\footnotetext{
$\leftarrow$

(Pro, proline-rich; WH2, WASP homology 2 domain; blue framing marks Cobl homology domain; and asterisks mark positions of KrRAPpPP motifs), and of (obl fragments used in this figure (Cobl fragments binding to Abp1 are in green, all others are in red). $\boldsymbol{D}, \boldsymbol{E}$, Cobl interacts with Abp 1 in an SH3 domain-dependent manner in yeast-2-hybrid analyses. Cobl bait plasmids led to strong reporter gene activity when combined with Abp1 full-length as a prey, whereas combination with an Abp1 mutant prey lacking the $\mathrm{SH} 3$ domain as well as combination of Abp1 with the control vector expressing only the DNA-binding domain did not sustain growth and reporter gene activity. Growth on quadruple drop-out plates (-AHLW) of normal and of 3-ATenhanced stringency $(\boldsymbol{D})$ as well as $\beta$-galactosidase ( $\beta$-Gal) activity $(\boldsymbol{E})$ were analyzed. $\boldsymbol{F}, \boldsymbol{G}$ Coprecipitation analyses of Abp1 SH3 domain with GFP-Cobl fragments, spanning the entire Cobl protein. Anti-GFP immunoblot analyses of cell lysates $(\boldsymbol{F})$ and coprecipitates $(\boldsymbol{G})$ reveal that Abp 1 interacts with the N-terminal third of Cobl. $\boldsymbol{H}, \boldsymbol{I}$, Coprecipitation analyses with the Abp1 $\mathrm{SH} 3$ domain reveal that Abp 1 binding is mediated by the second proline-rich domain but not by the KrRAPpPP motifs of the Cobl homology domain. $J-L$, Coprecipitation analyses of the $\mathrm{N}$-terminus of Cobl with immobilized wild-type Abp1 $\mathrm{SH} 3$ domain, an SH3 mutant (SH3 ${ }^{\text {mut }}$ ), and GST, respectively, show that the Cobl/Abp1 interaction is a classical SH3 domain/PxxP interaction.
} 

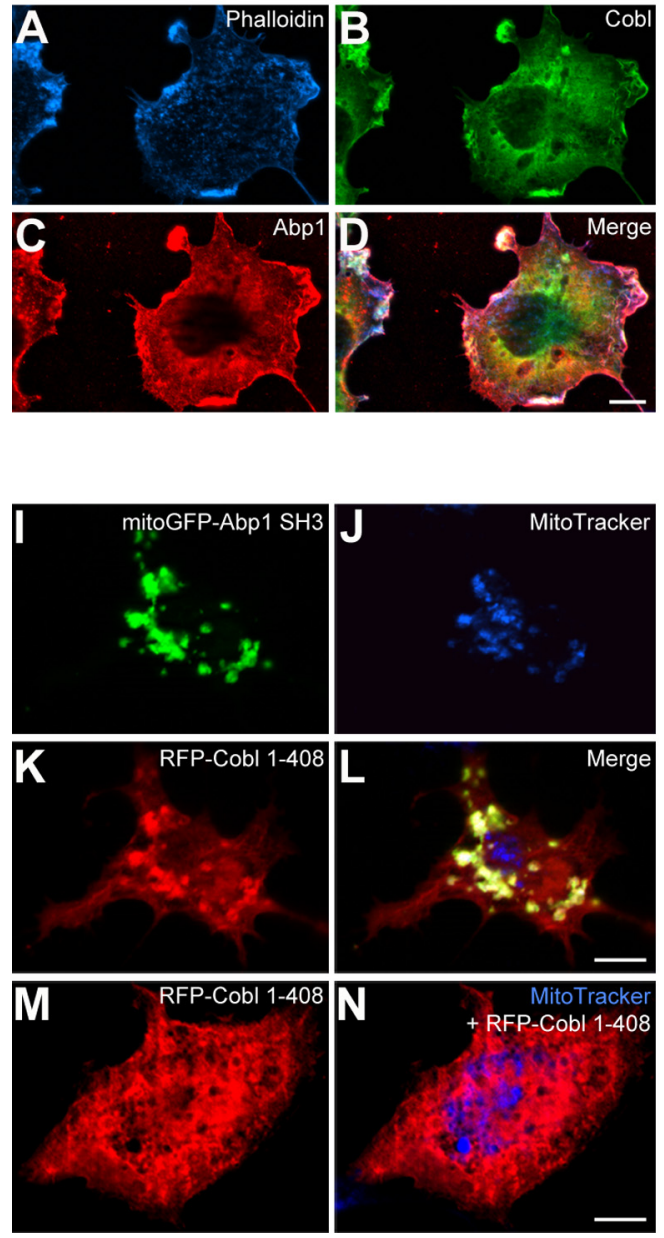

Figure 4. Abp1 associates with the N-terminus of Cobl in vivo. $\boldsymbol{A}-\boldsymbol{D}$, Colocalization of F-actin ( $\boldsymbol{A}$, phalloidin), GFP-Cobl (B), and myc-Abp1 (C) in COS-7 cells. Predominantly at F-actin-rich ruffles, colocalization appears white in the merged image (D). $\boldsymbol{E}-\boldsymbol{H}$, Heterologous coimmunoprecipitation of GFP-tagged Cobl $1-408(\boldsymbol{H})$ with Flag-Abp1 (IP; $\boldsymbol{G})$ expressed in HEK293 cells (lysates, $\boldsymbol{E}, \boldsymbol{F})$. I- $\boldsymbol{L}$, COS-7 cells expressing mitoGFP-Abp1 SH3 domain at the outer mitochondrial membranes ( $\boldsymbol{I}$ ) show a recruitment of RFP-Cobl $1-408$ (K) to mitochondria ( $\boldsymbol{J}$, MitoTracker). Colocalization appears

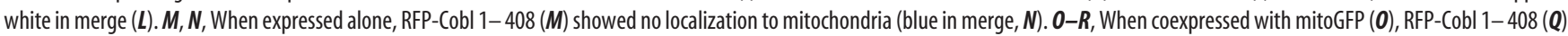
showed no colocalization with mitoGFP-coated, MitoTracker-stained $(\boldsymbol{P})$ mitochondria, as seen in the merged image $(\boldsymbol{R})$. Likewise, mitoGFP-Abp1 SH3 ( $\boldsymbol{S})$ did not recruit RFP (U) to mitochondria $(\boldsymbol{T})$, as seen in the merge (V). Thus, the Cobl/Abp1 interaction specifically occurred between the Cobl and Abp1 moieties of the fusion proteins. Scale bars: $10 \mu \mathrm{m}$.

3C) and Abp1 full-length fusion proteins led to robust reporter gene activity. In contrast, offering an Abp1 deletion mutant lacking the SH3 domain did not lead to activation of the reporter systems when combined with the Cobl bait. Thus, the Cobl/Abp1 interaction is dependent on the Abp1 SH3 domain located at the C-terminus of Abp1 (Fig. 3B-E).

Coprecipitation experiments with immobilized GST-Abp1 $\mathrm{SH} 3$ domain and a panel of GFP-Cobl fusion proteins spanning the entire length of Cobl showed that the interaction is mediated by the Cobl N-terminus and that the Abp1 SH3 domain is not only crucial but also sufficient for interaction (Fig. $3 F, G$ ). Cobl 1-408 was specifically and effectively precipitated. Cobl 1-408 contains the so-called Cobl homology domain of Cobl (Pfam Database accession number PF09469). In contrast, all Cobl constructs comprising more C-terminal regions of Cobl, including the actin-nucleating C-terminal domain of Cobl with the proline-rich linker L2 found to be crucial for actin nucleation (Ahuja et al., 2007), did not interact with Abp1 (Fig. 3 F, G). Also the KrRAPpPP-motifs that define Cobl homology domains and that were shown to be interaction sites for syndapin I (Schwintzer et al., 2011) did not associate with the Abp1 SH3 domain. In contrast, a Cobl fragment encompassing the second proline-rich domain (Pro) (amino acids 319-473) bound to the Abp1 SH3 domain (Fig. $3 H, I$ ). In line with the proline-rich nature of the Abp1-binding area, further experiments revealed that the Cobl/ Abp1 interaction is based on a classical SH3 domain/PxxP interaction, as mutations of glycine 422 and proline 425 within the Abp1 SH3 domain effectively disrupted the Cobl/Abp1 association (Fig. 3J-L).

\section{Cobl interacts with Abp1 in vivo}

Immunofluorescence analyses using an Apotome revealed that Cobl and Abp1 coexpressed in COS-7 cells colocalize at the F-actin-rich and ruffling leading edge (Fig. $4 A-D$ ). Since both Abp1 and Cobl were shown to colocalize with F-actin (Kessels et al., 2000; Ahuja et al., 2007), it was possible that this colocalization may represent a mere coincidence. By heterologous coimmunoprecipitation studies, we therefore next addressed complex formation of Cobl with Abp1. GFP-Cobl 1-408 was successfully coimmunoprecipitated with Flag-Abp1 from HEK293 cell lysates (Fig. $4 E-H$ ). Thus, Cobl/Abpl complexes can be formed in vivo. Since neither coexpressed GFP was precipitated by anti-Flag antibodies nor Abpl or Cobl proteins were found in immunoprecipi- 

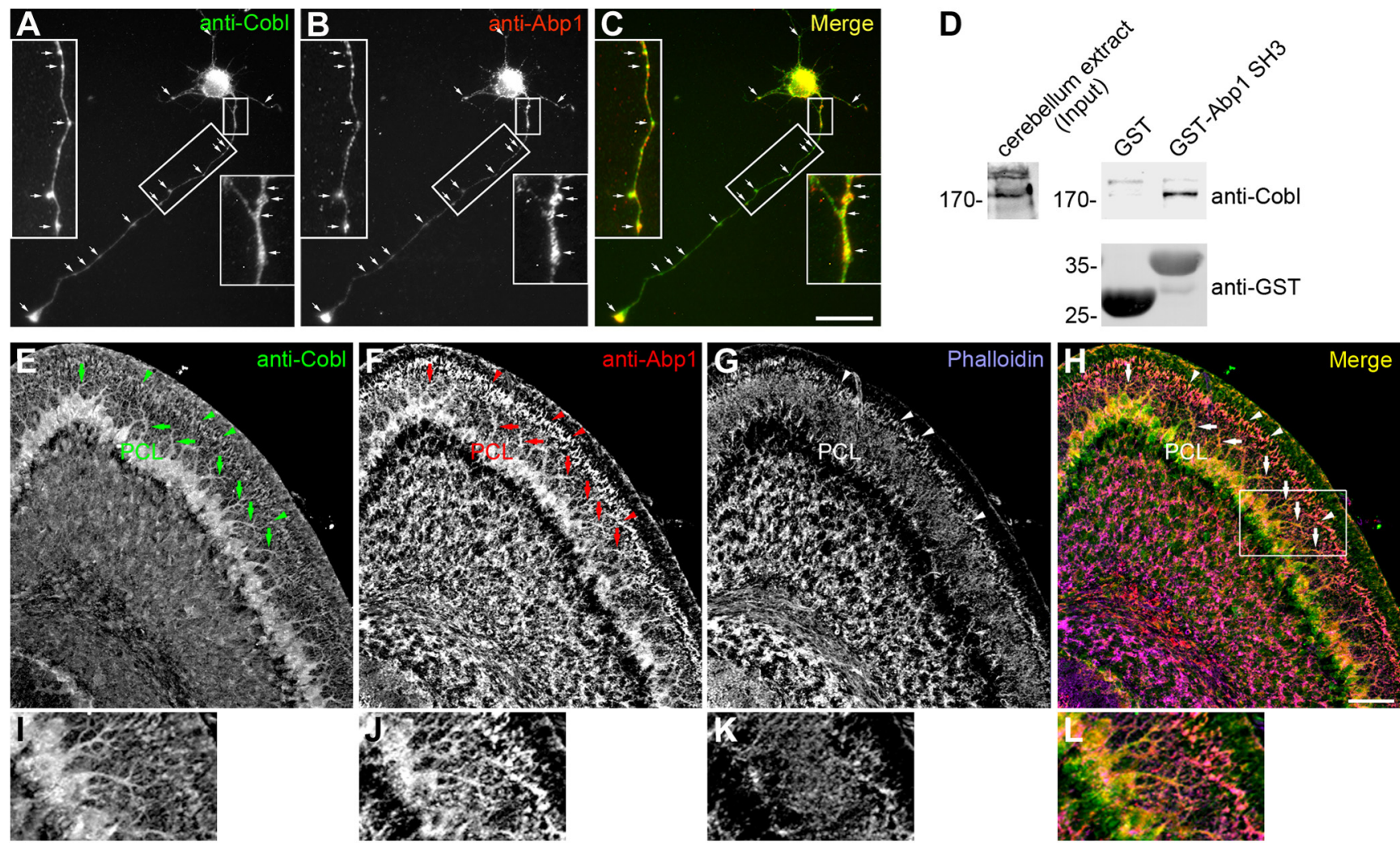

Figure 5. The distribution of Cobl and Abp1 overlaps. $\boldsymbol{A}-\boldsymbol{C}, \boldsymbol{E}-\boldsymbol{L}$, Immunofluorescence examinations of the localization of Cobl using rabbit anti-Cobl ${ }^{\mathrm{DBY}}$ antibodies $(\boldsymbol{A}, \boldsymbol{E}, \boldsymbol{I})$ and of $A b p 1$ using guinea pig anti-Abp1 antibodies $(\boldsymbol{B}, \boldsymbol{F}, \boldsymbol{J})$ in dissociated primary hippocampal neurons at DIV $2(\boldsymbol{A}-\boldsymbol{C})$ and in cerebellar slices of 10-day-old mice $(\boldsymbol{E}-\boldsymbol{L})$. Spatial overlap of both proteins is highlighted in the merged images $(\boldsymbol{C}, \boldsymbol{H}, \boldsymbol{L})$. In dissociated neurons, colocalization was observed in the cell body, in neurites, in puncta in the axon, and in both axonal and dendritic growth cones (arrows in $A-C)$. Insets in $\boldsymbol{A}$-C show enlargements of boxed areas. Scale bar: C, $25 \mu \mathrm{m}$. D, Coprecipitation analyses with immobilized GST and GST-Abp 1 SH3 domain, respectively, revealed a specific interaction between endogenous Cobl from cerebellum and the GST-Abp1 SH3 domain. $\boldsymbol{E}-\boldsymbol{H}$, Cobl, Abp1, and phalloidin colocalized in the Purkinje cell layer (PCL) of P10 cerebella as well as in Purkinje cell dendrites extending into the molecular layer (arrows) and overlapped with F-actin-rich sites at distal regions of the molecular layer (arrowheads). Scale bar: $\boldsymbol{H}, 50 \mu \mathrm{m}$. $\boldsymbol{I}-\boldsymbol{L}$, Twofold magnification of the area boxed in $\boldsymbol{H}$.

tates with unrelated IgGs, the Abp1 interaction with GFP-Cobl $1-408$ was specific (Fig. $4 E-H$ ).

To further support that Cobl interacts with the Abp1 SH3 domain via its $\mathrm{N}$-terminal part in vivo and to firmly exclude putative postsolubilization artifacts, we targeted a GFP-tagged Abp1 SH3 domain to the outer membrane of the mitochondria of COS-7 cells. Correct targeting was confirmed by colocalization with MitoTracker (Fig. 4I-L,S,T). Coexpressed RFP-Cobl 1-408 clearly accumulated at Abp1 SH3 domain-coated mitochondrial membranes (Fig. 4I-L).

This shift in localization of Cobl 1-408 depended on the Abp1 SH3 domain attached to mitochondrial membranes because Cobl 1-408 did not localize to mitochondria when transfected alone (Fig. $4 M, N$ ) and it did not localize to mitochondria coated with GFP (Fig. 4O-R). Further control experiments demonstrated that the Abp1 SH3 domain did not recruit RFP (Fig. 4S$V)$. Thus, the interaction observed specifically occurred between Cobl 1-408 and the Abp1 SH3 domain in living cells and is strong enough to shift the localization of Cobl.

\section{The neuronal localization of Cobl and Abp1 overlaps in} developing hippocampal neurons and in Purkinje cells of the cerebellum

To address whether the endogenous Cobl and Abp1 proteins may indeed work together, we first turned to dissociated primary hippocampal neurons, which represent an established system for functional analyses of Abp1 (Pinyol et al., 2007) and Cobl (Ahuja et al., 2007). Colocalization studies showed that both Abp1 and Cobl were present in the entire cell with accumulations in the cell body and in growth cones of cells kept in culture for 2 days. Anti-Cobl-positive puncta, which were also immunopositive for Abp1, were observable in both the developing axon and in the dendrites (Fig. $5 A-C$, arrows).

Immunohistological examinations of brain sections from adult mice showed that, in line with the staining in dissociated hippocampal neurons, both Cobl and Abp1 displayed marked immunosignals in the dentate gyrus and in the different areas of the hippocampus (data not shown). Studies with rabbit antiCobl antibodies raised against the amino acids 175-324 (ARA) and 750-1005 (DBY), respectively, hereby yielded consistent results (for detailed characterization of the rabbit anti-Cobl antibodies used for these analyses see Schwintzer et al. (2011)).

Complex formation of Abp1 with endogenous Cobl from cerebella of P10 mice was addressed by coprecipitation. These experiments confirmed a specific, $\mathrm{SH} 3$ domain-mediated association of Abp1 with cerebellar Cobl (Fig. 5D).

To further address Cobl/Abp1 complex formation, we incubated cerebellar sections from mice at P10 with antibodies against both proteins and fluorescently labeled phalloidin. In parallel, incubations with secondary antibody alone and control experiments with unrelated antibodies affinity-purified from the same antisera as the anti-Cobl antibodies used were done to control for the specificity of the immunolabeling. Whereas granule cells were only immunopositive for Abp1, Purkinje cells showed an obvious 


\author{
Antibody \\ characterization
}

A

$\mathbf{A}_{130}$

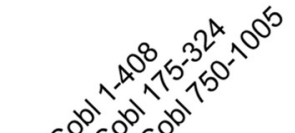

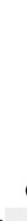

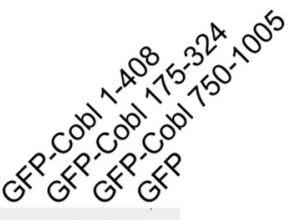

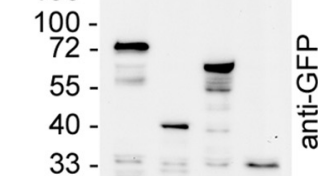

C

$24-$

130

D 130

$B_{130}$

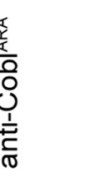

24 -

$100-$

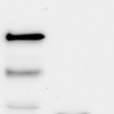

100 -

72 - - 72

$55--\quad \frac{0}{0}$
$40-\longrightarrow \frac{1}{0}$
$33-\square$

24 -
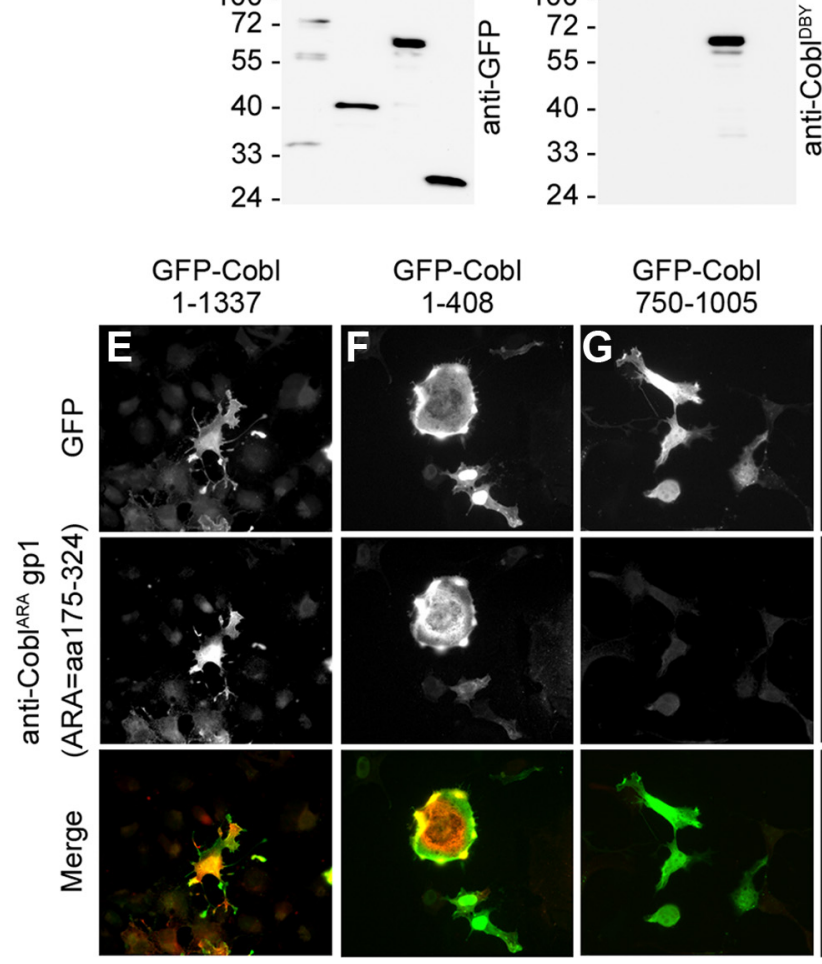

GFP-Cobl

$1-408$
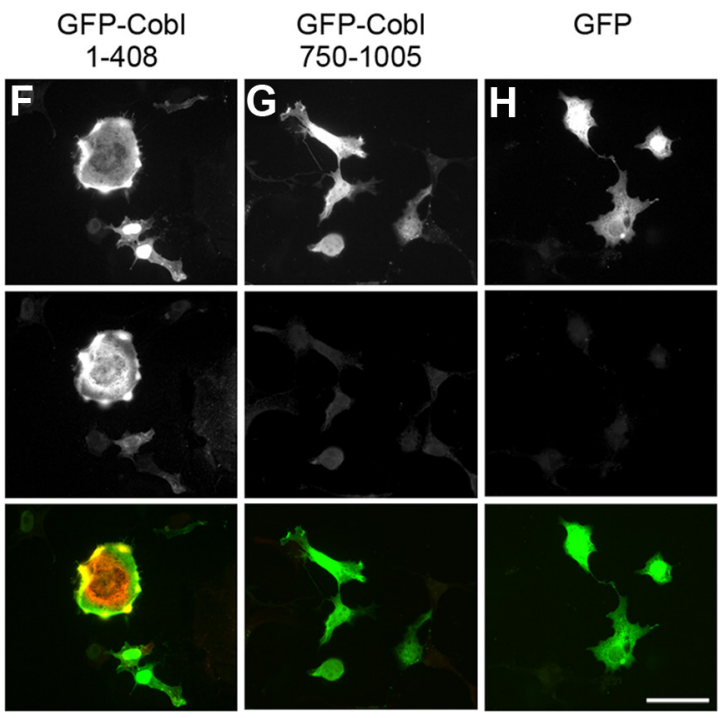

\section{Subcellular fractionation}

$$
\text { I }
$$

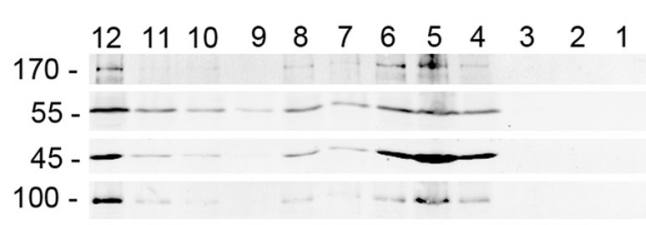

\section{Cobl \\ Abp1 \\ Actin \\ Insulin receptor tyrosine kinase}

\section{ColP of endogenous proteins}
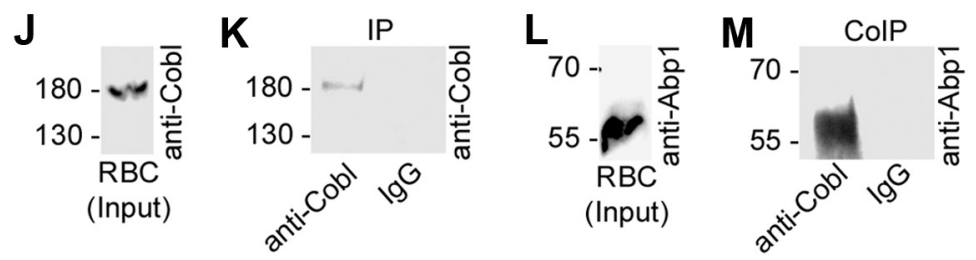

Figure 6. Cobl/Abp1 protein complexes exist in the brain. $A-D$, Characterization of guinea pig anti-Cobl ${ }^{A R A}$ antibodies raised against Cobl amino acids $175-324(\boldsymbol{B})$ and anti-Cobl ${ }^{\mathrm{DBY}}$ antibodies raised against Cobl amino acids $750-1005$ (D) by immunoblot analyses using GFP-tagged Cobl deletion mutants including and lacking the epitopes of the antibodies, respectively. Cobl deletion mutants were detected by anti-GFP immunoblotting $(\boldsymbol{A}, \boldsymbol{C})$. $\boldsymbol{E}-\boldsymbol{H}$, Characterization of the guinea pig anti-Cobl ${ }^{\text {ARA }}$ antibodies in immunofluorescence experiments with COS-7 cells transfected with the indicated GFP-Cobl constructs and GFP, respectively (top , green in merges). Immunostainings obtained with anti-Cobl antibodies are shown in the middle (red in merges). Guinea pig enrichment and colocalization of both Cobl and Abp1 (Fig. 5E-L).

In addition to the cell bodies in the Purkinje cell layer, also the dendritic arbors (arrows) as well as the peripheral border of the molecular layer (arrowheads) were marked by colocalization of Cobl and Abp1 (Fig. $5 E-L)$. Abp1 thus colocalized with Cobl in Purkinje cell dendrites.

Endogenous Cobl and Abp1 copurify with cell cortex components and form Cobl/Abp1 protein complexes in the brain

A common function for Cobl and Abp1 in neuronal morphogenesis would require both proteins to associate in the brain and to localize to the cell cortex to bring about cell shape changes. To test this, we raised guinea pig antibodies against both Cobl amino acids 175-324 (ARA) and Cobl amino acids 750-1005 (DBY). In Western blot analyses, both antibodies specifically recognized their antigens as GFP-fusion proteins equally well as anti-GFP antibodies but did not detect Cobl-fusion proteins lacking the antigenic region (Fig. $6 A-D)$.

Characterizations of both antibodies by immunofluorescence analyses of overexpressed GFP-Cobl and deletion mutants thereof showed that both antibodies were also suitable for detection of Cobl in cells, as they detected Cobl full-length and deletion mutants containing their antigenic region but not other parts of $\mathrm{Cobl}$ fused to GFP (Fig. $6 E-H$; data not shown for guinea pig anti-Cobl ${ }^{\mathrm{DBY}}$ ).

To address a common function of Cobl and Abp1 in neuronal morphogene-

\section{$\leftarrow$}

anti-Cobl ${ }^{\text {ARA }}$ antibodies specifically recognized the cells transfected with antigen-containing GFP-Cobl constructs $(\boldsymbol{E}, \boldsymbol{F}$; yellow in merges), whereas cells expressing GFP-Cobl fragments, which do not contain the antigenic region of the respective antibody $(\boldsymbol{G})$ or cells that just expressed GFP $(\boldsymbol{H})$, as well as untransfected cells (stained using fluorescently labeled phalloidin; data not shown) did not show any anti-Cobl immunosignals. Scale bar, $50 \mu \mathrm{m}$. I, Density gradient centrifugation of brain extracts analyzed by Western blotting using guinea pig anti-Cobl ${ }^{\mathrm{DBY}}$ and anti-Abp1 antibodies show that both proteins cofractionate. Note that fractions $4-6$ correspond to floating plasma membrane material, whereas fraction 12 represents material remaining at the bottom of the gradient. Insulin receptor tyrosine kinase was used as plasma membrane marker. J-M, Coimmunoprecipitation of endogenous Cobl/ Abp1 complexes from rat brain cytosol (RBC) using anti-Cobl antibodies and anti-Abp 1 antibodies, respectively (Input; $J, L)$. Cobl was specifically immunoprecipitated by immobilized guinea pig anti-Cobl ${ }^{\text {ARA }}$ antibodies (IP; $\left.\boldsymbol{K}\right)$. Analyses of immunoprecipitates with rabbit anti-Abp1 antibodies revealed a specific coimmunoprecipitation of Abp 1 with Cobl $(\mathrm{CoIP} ; M)$ 

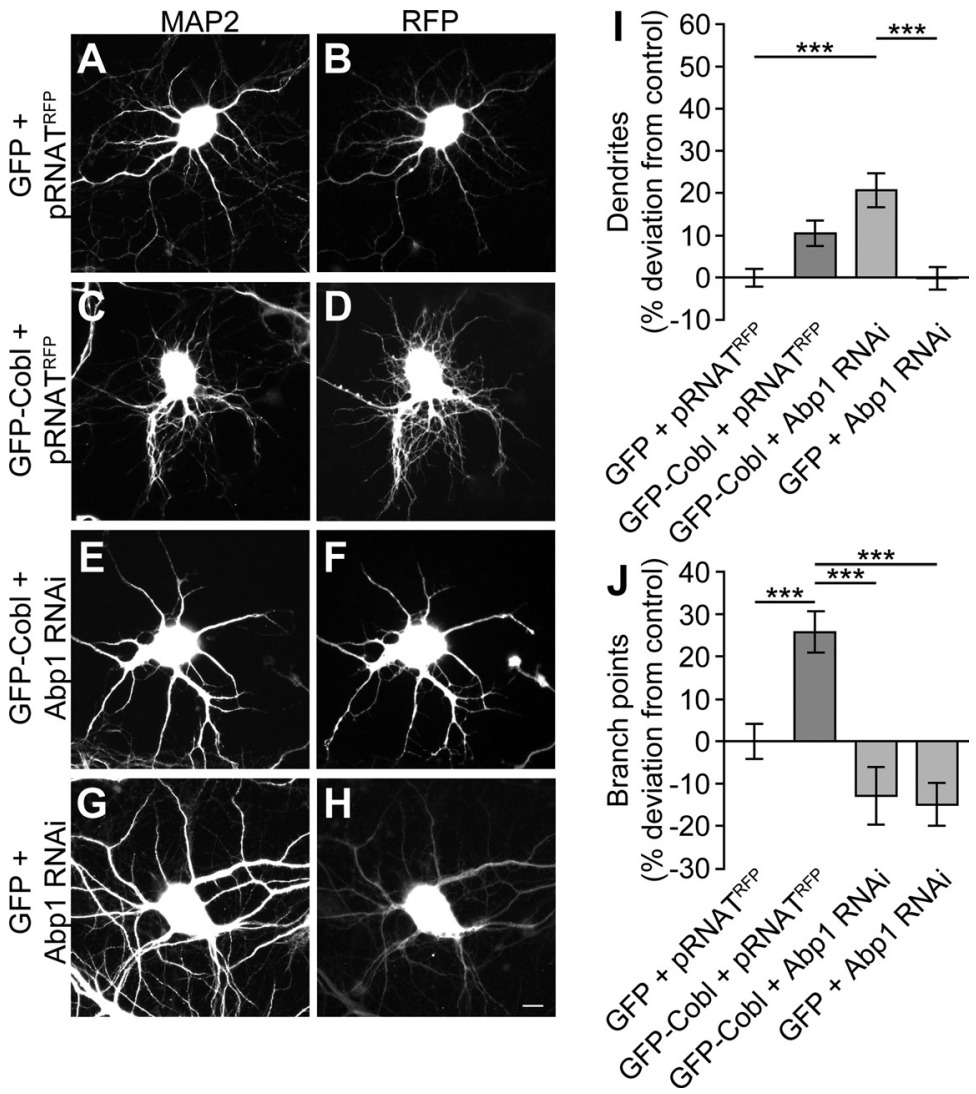

Figure 7. Cobl-mediated dendritic branching of dissociated hippocampal neurons critically depends on Abp1.A-H, Dissociated primary hippocampal neurons at DIV 4 were transfected as indicated and imaged $2 \mathrm{~d}$ later. Staining of endogenous MAP2 was used as a transfection-independent tracer to evaluate the morphology of transfected neuronal cells $(\boldsymbol{A}, \boldsymbol{C}, \boldsymbol{E}, \boldsymbol{G})$. RFP reporter signals from RNAi plasmids (pRNAT) are shown in $\boldsymbol{B}, \boldsymbol{D}, \boldsymbol{F}$, and $\boldsymbol{H}$. Scale bar, $10 \mu \mathrm{m}$. Compared with control, i.e., neurons cotransfected with GFP and an empty pRNAT ${ }^{\mathrm{RFP}}(\boldsymbol{A}, \boldsymbol{B})$, cells cotransfected with GFP-Cobl and pRNAT ${ }^{\mathrm{RFP}}(\boldsymbol{C}, \boldsymbol{D})$ showed a trend toward a Cobl-mediated increase in the numbers of dendrites $(\boldsymbol{I})$ and a highly significant increase in the number of dendritic branching points $(\boldsymbol{J})$. Note that cotransfection of GFP-Cobl and established RNAi tools against Abp1 (Abp1 RNAi) $(\boldsymbol{E}, \boldsymbol{F})$ revealed that the Cobl-mediated increase in dendritic branching crucially depends on $\operatorname{Abp1}(\boldsymbol{J}) . n=88-159$ neurons. Error bars indicate SEM. ${ }^{* * *} p<0.001$.

sis, we first conducted subcellular fractionations. These analyses showed that a portion of Cobl and of Abp1 indeed copurified and that both proteins floated up with plasma membrane material purified according to Kretzschmar et al. (1996). Immunodetection of actin in the same sucrose gradient fractions showed that at least some structures of the cortical actin cytoskeleton are still attached to the plasma membrane material (Fig. $6 I$ ).

To finally examine whether the endogenous proteins indeed form complexes in the brain, we used the new guinea pig anti-Cobl antibodies raised using a region of Cobl, which did not overlap with the Abp1 SH3 domain-binding region, in coimmunoprecipitation analyses from rat brain extracts (Fig. $6 J-M$ ). Anti-Cobl immunoblotting demonstrated that guinea pig anti-Cobl ${ }^{\mathrm{ARA}}$ antibodies were able to immunoprecipitate endogenous Cobl from rat brain lysates (Fig. $6 \mathrm{~K}$ ). Importantly, endogenous Abp1 was specifically coimmunoprecipitated with Cobl (Fig. 6M).

\footnotetext{
Abp1 is important for Cobl-mediated dendrite branching Cobl-mediated actin nucleation is important for proper dendritic development of dissociated hippocampal neurons (Ahuja et al., 2007). The only known roles of Abp1 in actin-mediated neuronal morphogenesis concern Arp2/3 complex- and N-WASP-dependent processes during axon development (Pinyol et al., 2007; Dharmalingam et al., 2009) and ProSAP/Shank-dependent organization of postsynaptic spines (Qualmann et al., 2004; Haeckel et al., 2008).
}

This raised the question whether Coblmediated dendritogenesis processes may critically rely on Abp1 at all. In line with previous observations in single transfections (Ahuja et al., 2007), also in double transfections with an RNAi vector expressing RFP as reporter ( $\mathrm{pRNAT}{ }^{\mathrm{RFP}}$ ), Cobl led to increased dendritic arborization compared with control cells expressing GFP and pRNAT ${ }^{\mathrm{RFP}}$ (Fig. $7 A-D, I, J)$. Interestingly, whereas Abp1 RNAi alone did not cause any significant impairments in dendritogenesis in the time frame investigated, Cobl-mediated dendrite branching was completely suppressed upon Abp1 RNAi (Fig. 7E-H).

Quantitative examinations revealed that the dendritic branch points of GFPCobl expressing neurons cotransfected with Abp1 RNAi were not distinguishable from cells expressing GFP and Abp1 RNAi. Abp1 RNAi thus completely abrogated this Cobl phenotype (Fig. 7J).

\section{Abp1 RNAi phenocopies Cobl} deficiency in cerebellar development Our examinations show that Cobl interacts with Abp1 in vitro and in vivo and that Abp1 is crucial for Cobl-mediated shaping of dissociated hippocampal neurons. Cobl plays a pivotal role in the differentiation of Purkinje cells in the developing cerebellum (Fig. 2). Abp1 is highly expressed in the cerebellum throughout development and its localization overlaps with that of Cobl in the cerebellum. We thus next followed the working hypothesis that, with Abp1, we have identified a molecular component that helps steer the Cobl actin nucleation machinery during Purkinje cell development.

If so, Abp1 loss-of-function phenotypes in Purkinje cells should resemble the Cobl loss-of-function phenotypes. Indeed, Abp1-deficient Purkinje cells showed an impaired P10-P12 differentiation. As observed for Cobl depletion, Abp1 RNAi led to less arborized Purkinje cells (Fig. 8A,B). Quantitative examinations showed that dendritic branch point numbers were highly significantly reduced in Purkinje cells subjected to Abp1 RNAi $(-62.3 \%$ when compared with the corresponding scrambled RNAi control) (Fig. 8G). Furthermore, the density of dendritic branch points was significantly reduced in Abp1-deficient cells $(-44.8 \%$; Fig. $8 H)$. Quantitative comparison with the Cobl lossof-function phenotypes showed that Abp1 and Cobl loss-offunction led to almost identical effects (Fig. $8 D-H$ ).

Furthermore, provided that Cobl and Abpl operate in one pathway, phenotypes resulting from double-RNAi (if efficient) should not differ much from the individual phenotypes. To address this question we constructed a pRNAT derivative, which drives the Cobl RNAi via a different promoter (U6.1 instead of H1.1) to avoid reduction of shRNA transcription upon double transfection of two RNAi vectors competing for the same endogenous factors. Individually, Cobl RNAi (U6.1) phenotypes led to phenotypes that were identical to Cobl RNAi (H1.1) (compare Fig. 2) and to Abp1 loss-of-function (Fig. 8A-H). 


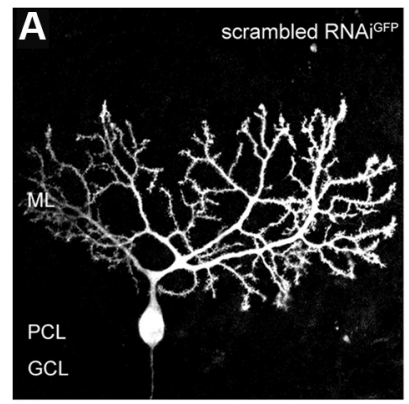

D
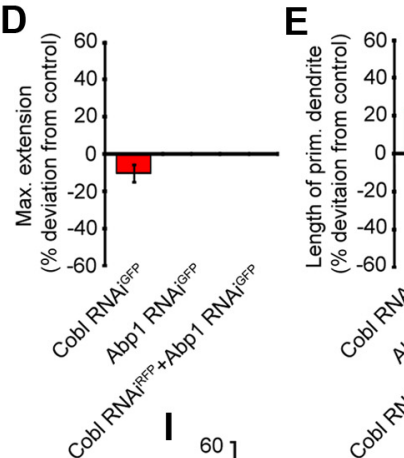

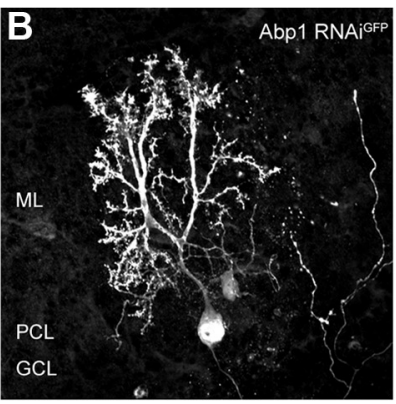

$\mathbf{F}$

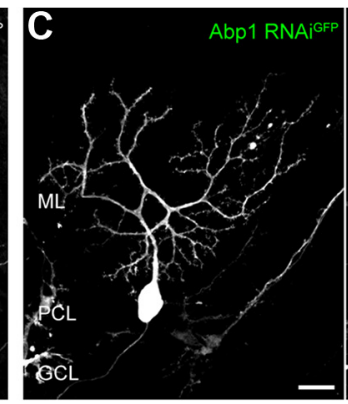

G

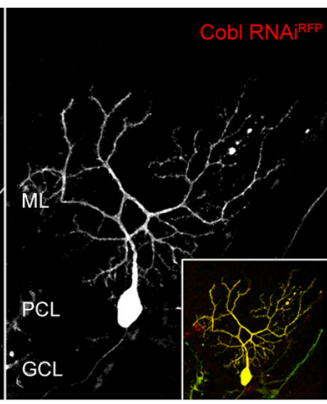

H
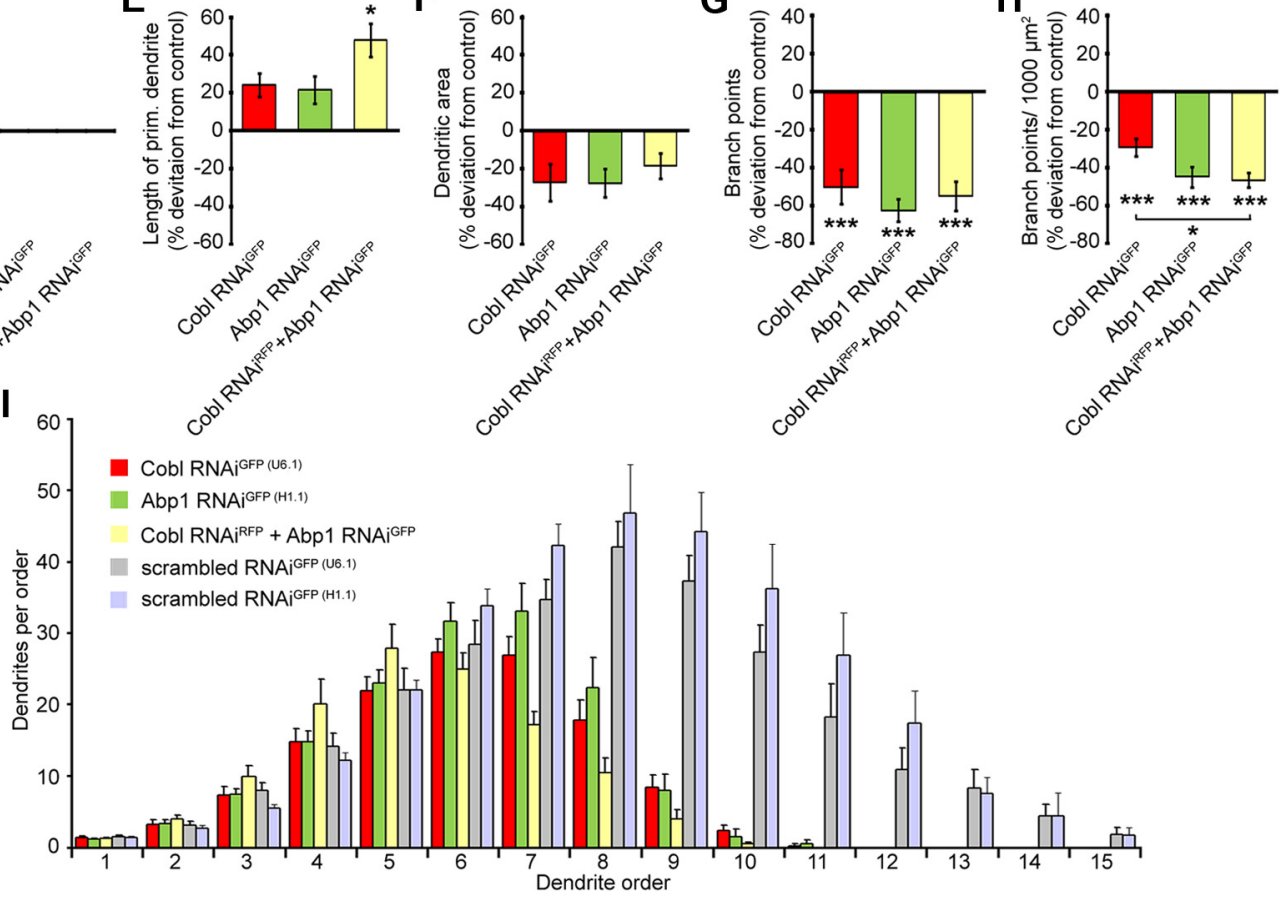

Figure 8. Postnatal development of cerebellar Purkinje cells is affected by Cobl and Abp 1 deficiency in a similar manner. $A-C$, Parasagittal, 250- $\mu \mathrm{m}$-thick cerebellar slices bombarded with scrambled RNAi ${ }^{\text {GFP }}$-coated gold bullets $(\boldsymbol{A})$, with Abp1 RNAi ${ }^{\text {GFP }}(\boldsymbol{B})$ and $\mathrm{Cobl}$ RNAi ${ }^{\mathrm{RFP}}$ in combination with Abp1 RNAi ${ }^{\text {GFP }}(\boldsymbol{C})$ at P10 and fixed $2 \mathrm{~d}$ later. Designation of cerebellar layers is based on accompanying DAPI staining (data not shown for clarity). Labeling of layers as in Figure 1. Scale bar, $20 \mu \mathrm{m}$. D-I, Quantitative evaluations of morphological parameters of Purkinje cells in cerebellar slice cultures transfected with Abp1 RNAi (pRNAT H1.1) $(n=36)$ and Cobl RNAi (pRNAT U6.1) $(n=35)$ vectors and transfected with both $(n=29)$, respectively, compared with control cells (scrambled RNAi ${ }^{\text {GFP }}$ in pRNAT U6.1 (for Cobl RNAi and Cobl RNAi/Abp1 RNAi) and H1.1, respectively; $n=27$ and $n=34$, respectively). Cobl RNAi phenotypes driven by the U6.1 promoter are similar to those seen with H1.1 (Fig. 2). Note that the Abp 1 loss-of-function phenotypes are virtually identical to those caused by Cobl RNAi. Error bars indicate SEM. ANOVA + Tukey's HSD post-test. ${ }^{*} p<0.05 ;{ }^{* * *} p<0.001$. I, Dendritic order analyses showing the loss of complexity of Abp1-, Cobl-, and Abp1- + Cobl-deficient Purkinje cell arbors. Data for scrambled RNAi ${ }^{\text {GFP (U6.1) }}$ and scrambled RNAi ${ }^{\text {GFP }(\mathrm{H1} 1.1)}$ controls are not significantly different in any order. Cobl RNAi ${ }^{\text {GFP }(U 6.1)}$ versus scrambled RNAi $^{\text {GFP }}{ }^{(U 6.1)}, p<0.001$ for dendrite orders $8-13, p<0.05$ for dendrite order 14. Abp1 RNAi $^{\text {GFP }}\left(\right.$ H1.1) versus scrambled RNAi ${ }^{\text {GFP }(H 1.1)}, p<0.01$ for dendrite order 8 and $13, p<0.001$ for dendrite orders $9-12$. Abp1 RNAi ${ }^{\text {GFP }}+$ Cobl RNAi ${ }^{\text {RFP }}$ versus scrambled RNAi ${ }^{\text {GFP }}{ }^{\text {U6.1) }}, p<0.01$ for order $7, p<0.001$ for orders $8-13$ and $p<0.05$ for order 14 .

When Cobl RNAi and Abp1 RNAi were combined, this led to defects very similar to those of the individual RNAis (Fig. $8 B-H$ ). Together, these data suggested that the interaction partners Cobl and Abp1 indeed work in one common pathway and that both proteins are crucial for the molecular pathway underlying Purkinje cell arborization.

Particularly higher order branching is defective in Cobl- and Abp1-deficient Purkinje cells

We next evaluated the branching defects seen upon Cobl RNAi, Abp1 RNAi, and Cobl RNAi + Abp1 RNAi in more detail. Careful quantitative analyses of the dendrite order of Cobl and Abp1 loss-of-function and of control Purkinje cells showed that specifically dendrites of higher order were affected. The analyses revealed a significant reduction in the number of dendrites of the orders 8-14 in Cobl RNAi cells (Fig. 8I).

Also in the analysis of dendrite orders, the Abp1 loss-of- function phenotype was very similar compared with that caused by Cobl loss-of-function. Especially higher order arborization was strongly impaired. Statistically significant impairments of dendrite branches were observed from order 8 to 13 (Fig. 8I). Identical defects were observed in Purkinje cells lacking both Cobl and Abp1 (Fig. 8I). Together, these observations demonstrate that Cobl and Abp1 are crucial factors for proper development of the elaborate cell morphologies of Purkinje cells.

\section{Cobl-mediated Purkinje cell dendritogenesis requires the} formation of Cobl/Abp1 complexes

Our data suggest that association of Cobl and Abp1 in one protein complex is a key requirement for proper Purkinje cell development and that Abp1 uses its affinity for newly polymerized, dynamic F-actin (Kessels et al., 2000) to bring about the cell biological functions of Cobl/Abp1 complexes (Fig. 9A). We addressed both of these mechanistical hypotheses by functional 
A

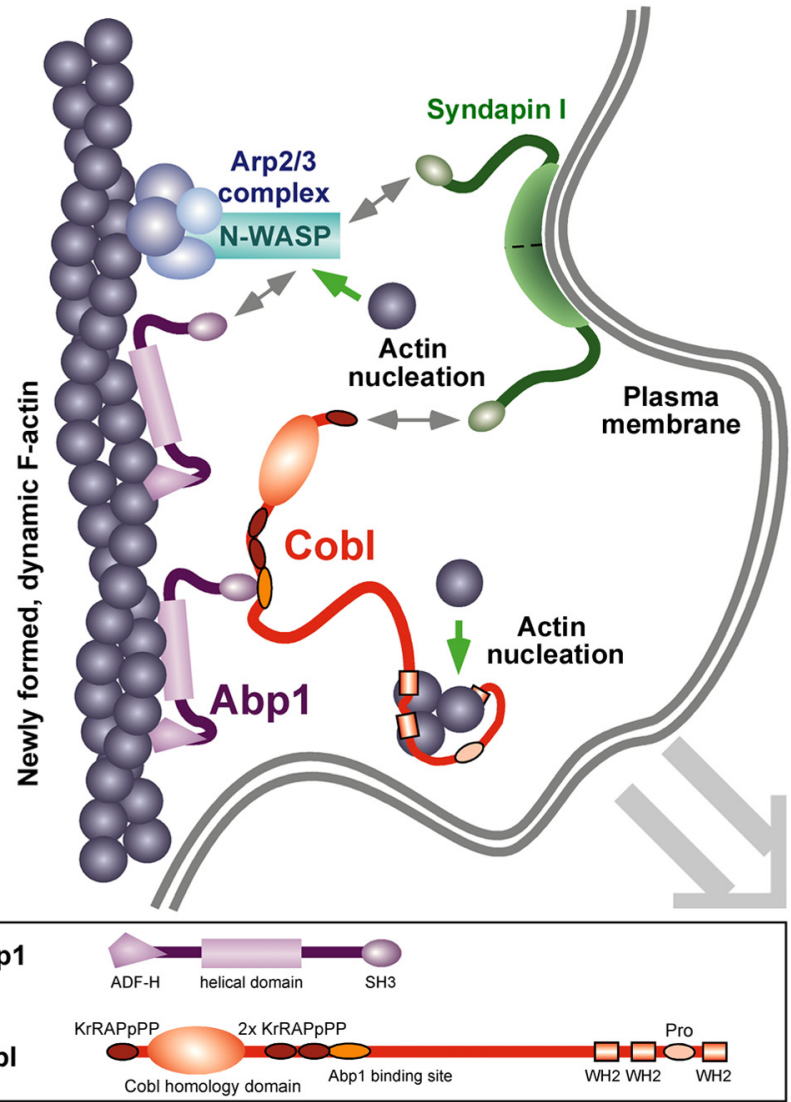

B

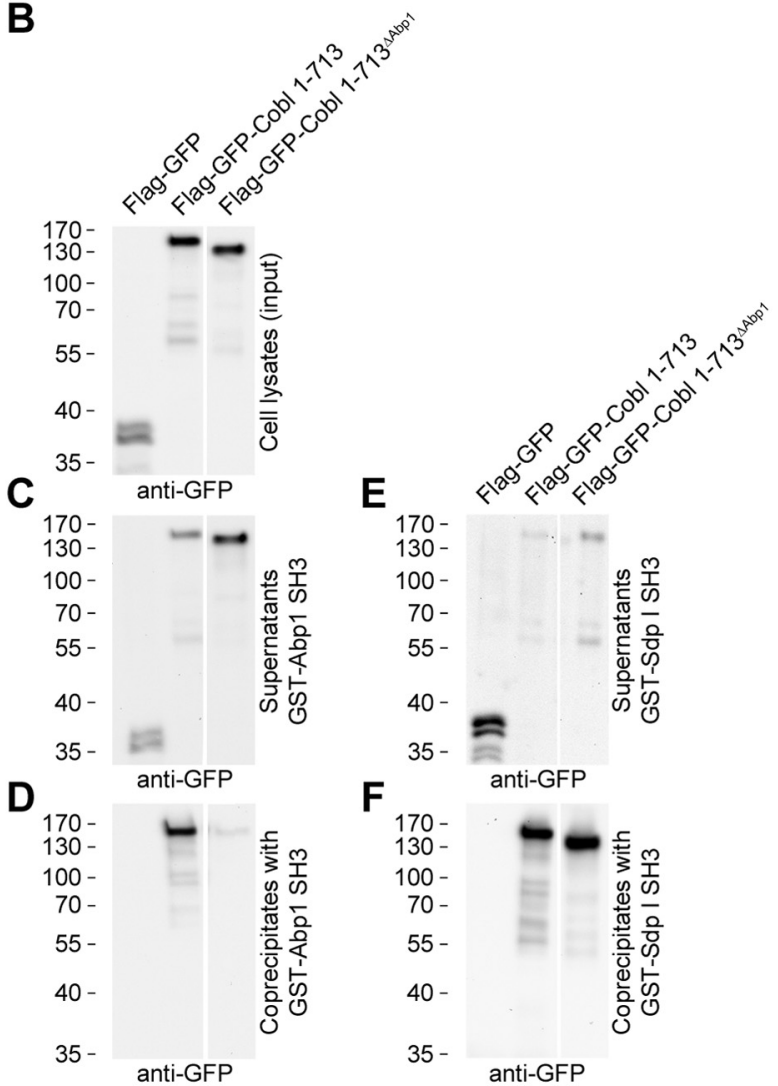

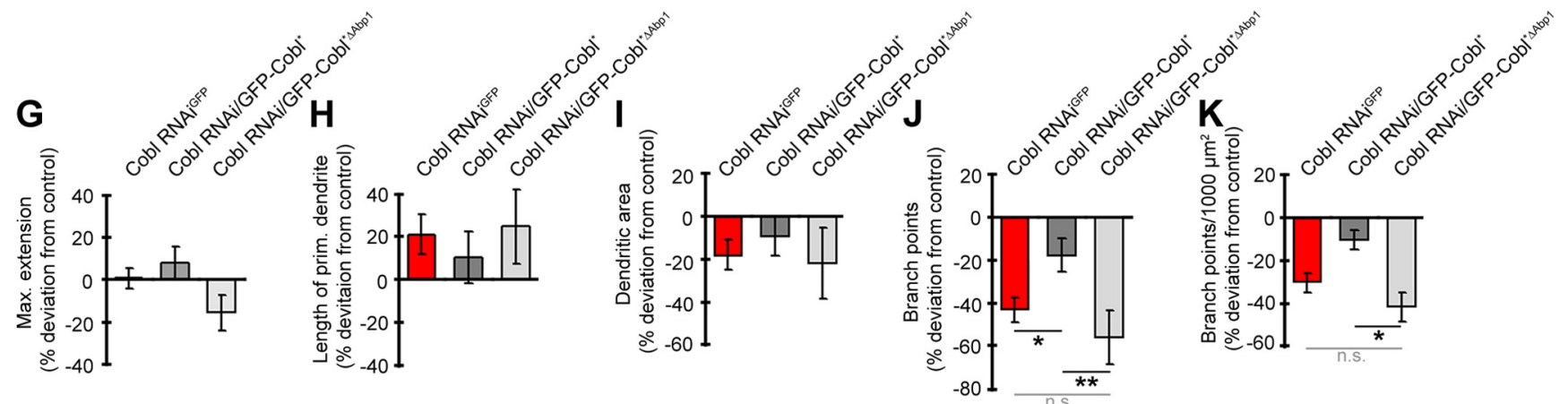

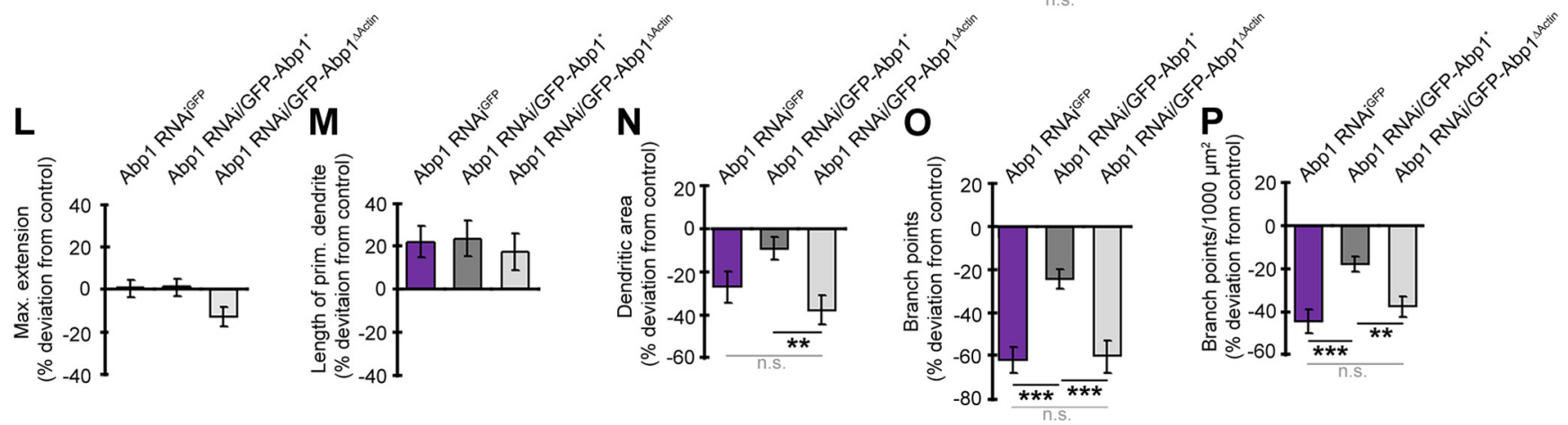

Figure 9. Functional Cobl/Abp1 complexes are crucial for Purkinje cell arborization. $A$, Model highlighting the molecular basis for the importance of Cobl/Abp1 complexes for proper differentiation of Purkinje cells embedded in the cerebellar architecture. Using its $\mathrm{N}$-terminal F-actin-binding modules Abp 1 can attach to dynamic actin filaments and recruit the actin nucleator Cobl. This leads to more actin polymerization and further recruitment of Abp 1 resulting in further recruitment of Abp 1 SH3 domain-binding nucleation promoting factors (either Cobl or N-WASP/Arp2/3 complex) providing a powerful feed-forward mechanism. This Abp1-mediated mechanism is complemented by syndapin I-mediated plasma membrane curvature sensing and physical interconnection of the Cobl and Arp2/3 complex machineries for actin nucleation. $B$, Coprecipitation analyses of GFP-Cobl 1-713 and of a $\Delta 8-28, \Delta 369-402$ mutant thereof (Cobl 1-713 ${ }^{\Delta \text { Abp } 1}$ ) expressed in HEK293 cells $(\boldsymbol{B})$ and incubated with immobilized GST-Abp1 SH3 $(\boldsymbol{C}, \boldsymbol{D})$ and GST-syndapin I SH3 $(\boldsymbol{E}, \boldsymbol{F})$. Supernatants $(\boldsymbol{C}, \boldsymbol{E})$ and eluates $(\boldsymbol{D}, \boldsymbol{F})$ from both experiments were immunoblotted with anti-GFP antibodies. Note that $\mathrm{Cobl} 1-713^{\triangle \mathrm{Abp} 1}$, which still binds to syndapin I $(\boldsymbol{F})$, is not able to associate with Abp1 anymore $(\boldsymbol{D})$. White lines represent lanes of the blots that were omitted for the purpose of this figure. G-P, Quantification of morphometric parameters of Purkinje cell arborization in cerebellar slice cultures. G-K, Coexpression of Cobl RNAi/GFP-Cobl ${ }^{* \Delta A b p 1}$ failed to rescue the observed reduction of branch points and branch point density, whereas re-expressing full-length Cobl (GFP-Cobl*) rescued all Cobl loss-of-function phenotypes. (Figure legend continues.) 
analyses. Cobl and Abp1 mutants lacking these properties should fail to restore Cobl and Abp1 functions in Cobl- and Abp1deficient Purkinje cells, respectively. First, we pinpointed the Abp1 binding sites in the Cobl homology domain of Cobl in more detail by coprecipitation studies with a variety of GFP-Cobl mutants (data not shown). Based on this work, we were finally able to create a Cobl mutant $\left(\mathrm{Cobl}^{\Delta \mathrm{Abpl}}\right)$ that is Abp1-binding-deficient, as demonstrated by coprecipitation experiments (Fig. $9 B-D$ ), yet does still associate with syndapin I, the only other Cobl homology domain-interacting protein known thus far (Fig. 9E, F).

We then incorporated RNAi-insensitive versions of Cobl $\left(\mathrm{GFP}^{-\mathrm{Cobl}^{*}}\right.$ ) and of $\mathrm{Cobl}^{\Delta \mathrm{Abpl}}\left(\mathrm{GFP}-\mathrm{Cobl}^{\star \Delta \mathrm{Abpl}}\right)$ into Cobl RNAi vectors and asked whether the ability of Cobl to associate with Abp 1 would be necessary to rescue the Cobl loss-of-function phenotypes on dendritic branching and branch point density. Quantitative and statistical analyses showed that re-expression of RNAi-insensitive Cobl successfully rescued both arborization phenotypes observed upon Cobl RNAi. Cobl RNAi-transfected Purkinje cells rescued by Cobl re-expression were indistinguishable from control (Fig. 9J,K).

In contrast, re-expression of the Abp1-binding-deficient Cobl mutant GFP-Cobl ${ }^{\star \Delta \text { Abpl }}$ did not affect the phenotypes that were caused by Cobl RNAi. Quantitative analyses showed that Cobl RNAi/GFP-Cobl ${ }^{\star \Delta \text { Abpl }}$ values were significantly different $(p<$ 0.01 and $<0.001$, respectively) from branch point numbers and densities observed upon rescue with full-length Cobl but indistinguishable from Cobl RNAi (Fig. 9G-K). These data strongly suggest that complex formation with Abp1 is a prerequisite for Cobl functions in Purkinje cell arborization.

\section{Abp1 functions in Cobl-mediated Purkinje cell dendritogenesis require $\mathrm{F}$-actin binding}

We next followed up on the hypothesis that in addition to direct complex formation Abpl's F-actin association is another key requirement for Cobl/Abp1 functions. For this purpose, we constructed Abp1 RNAi vectors, which either coexpress an RNAi-insensitive Abp1 protein (Abp1 RNAi/GFP-Abpl ${ }^{*}$ ) or a mutant that lacks the F-actinbinding ADF-H and the helical domain (Kessels et al., 2000) and therefore is F-actin binding deficient (Abp1 RNAi/GFP$A b p 1^{\Delta \text { Actin }}$ ). Overexpression of Abp1 (in scrambled RNAi vector) did not lead to phenotypes in any of the five parameters quantitatively analyzed (data not shown). Re-expression of wild-type Abp1 indeed successfully rescued the defects caused by Abp1 deficiency. Quantitative analyses showed that in addition to suppression of the dendritic area defects that, with $p<0.01$, were significantly different from control in this context (not indicated in Fig. 9N), dendritic branch point numbers and density impairments also observed upon Abp1 RNAi were significantly suppressed upon re-expression of RNAi-insensitive full-length Abp1 (Fig. 9L-P).

In contrast to the full-length $\mathrm{Abp} 1$ protein, re-expression of the $\mathrm{F}$-actin binding-deficient mutant Abp1 ${ }^{\Delta \text { Actin }}$ completely failed to rescue any of the Abp1 loss-of-function phenotypes. Dendrite branching of Purkinje cells coexpressing Abp1 ${ }^{\Delta \text { Actin }}$ together with Abp1 RNAi was indistinguishable from Abp1 RNAitransfected Purkinje cells (Fig. 9L-P). Alone, overexpression of

$\leftarrow$

(Figure legend continued.) Cotransfection of Abp1 RNAi GFP and an Abp1 mutant that lacks the F-actin ability of Abp1 (Abp1 RNAi/GFP-Abp1 ${ }^{\triangle A \text { Atin }} ; \Delta$ ad 1-281) failed to rescue the observed reduction of branch points and branch point density $(\boldsymbol{L}-\boldsymbol{P})$. Data are represented as mean \pm SEM. $\left({ }^{*} p<0.05\right.$, $\left.{ }^{* *} p<0.01,{ }^{* * *} p<0.001\right) ; n=34$ (scrambled RNAi ${ }^{\text {GPP }}$ ), $n=21$ (Cobl RNAi ${ }^{\text {GFP }}$ ), $n=10$ (Cobl RNAi/GFP-Cobl*), $n=9$ (Cobl RNAi/GFP-Cobl ${ }^{*} \triangle$ Abp1 $), n=36$ (Abp1 RNAi ${ }^{\text {GFP }}$ ), $n=26$ (Abp1 RNAi/ GFP-Abp1 $\left.^{*}\right), n=22$ (Abp1 RNAi/GFP-Abp1 ${ }^{\Delta \text { Actin }}$ ).
Abp1 ${ }^{\Delta \text { Actin }}$ did not lead to changes in branch point density (data not shown).

Together our functional rescue experiments highlight the fact that not only the individual proteins Cobl and Abpl but Cobl/ Abp1 complex formation is crucial for proper Purkinje cell development and that Cobl- and Abp1-mediated functions in bringing about the elaborate cell morphologies of Purkinje cells rely on the F-actin binding capabilities of Abp1.

\section{Discussion}

The basis for the function and astonishing abilities of vertebrate brains lies in the complex architecture of neuronal networks and the organized connectivity of neurons within neuronal arrays and layers. Microtubules and actin filaments are crucially involved in bringing about the morphological complexity neurons develop during their differentiation. Such cell shape changes predominantly occur in brains of embryos and newborns but to a lesser extent are life-long. Although these remodeling processes are an important basis for regeneration plasticity of the brain, astonishingly little is known about how actin filament formation giving rise to the forces initiating and promoting the complex morphologies of neuronal cells is brought about (Kessels et al., 2011). Among the few cellular machines generating actin filaments de novo that are known, the actin nucleator Cobl is predominantly expressed in the nervous system (Ahuja et al., 2007). Thus far, however, knowledge of the physiological functions of Cobl in the brain is still sparse.

We show that Cobl is crucial for proper arborization of Purkinje cells ensuring the high connectivity of neurons in the molecular layer of the cerebellum. Cobl-deficient Purkinje cells displayed a severely impaired dendritic branching that manifested in reduced absolute numbers of postnatally obtained dendritic branches, a reduced branch point density, and a severe lack of higher order branches. Cobl depletion thus led to fully extended, yet low-complexity Purkinje cell arbors. The importance of Cobl for postnatal development of the cerebellum was reflected by Cobl expression in Purkinje cells during early and later stages of cerebellar development. The Cobl loss-of-function phenotypes observed were specific because they were rescued by restoring the expression of Cobl in Purkinje cells by re-expressing RNAi-insensitive Cobl. Furthermore, branching defects were also seen in Cobl RNAi-transfected dissociated hippocampal neurons.

In addition to Cobl, whether other actin nucleators are crucially involved in Purkinje cell arborization is largely unknown. Thus far, to our knowledge, only the formin Delphilin has been characterized functionally in Purkinje cells. Delphilin interacts with the Purkinje cell-specific glutamate receptor delta 2 subunit (Miyagi et al., 2002). Gene knock-out of Delphilin in mice showed facilitated long-term depression, but no abnormalities were detected in cerebellar histology, Purkinje cell cytology, and synapse formation with parallel fibers (Takeuchi et al., 2008). In terms of Arp $2 / 3$ complex-mediated actin nucleation, it is known that at least one of its activators, WAVE-1, is expressed in Purkinje cells. The sensorimotor retardation observed in WAVE-1 knock-out mice may be attributable to some cerebellar malfunction (Soderling et al., 2003). Purkinje cells have also been reported to express Formin-2 and Spire-1, but their functional analysis is still pending (Schumacher et al., 2004).

In addition to the role in postnatal cerebellar development revealed in this study, Cobl seems to function in embryonic development. Gene trap experiments suggested that Cobl mRNA is present in a small group of cells in the gastrulating embryo giving 
rise to the axial midline (Gasca et al., 1995). The gene-trap allele had no identifiable phenotype by itself, as it seems to be a weak hypomorph, but it enhanced loop-tail defects in neural tube closure by an unknown mechanism. The loop-tail gene encodes VANGL2, a planar cell polarity pathway component (Carroll et al., 2003). In line, zebrafish Cobl was maternally expressed and Cobl morphant embryos exhibited failures in proper body axis formation during the first hours after fertilization. At day 3 after fertilization, Cobl morphant embryos displayed kidney cysts and dilated medial pronephric tubules (Ravanelli and Klingensmith, 2011).

The crucial role of Cobl in the postnatal differentiation of Purkinje cells suggests that cortical cytoskeletal functions of Cobl shape cells. We observed that Cobl was present at the cell cortex and physically interacts with $\mathrm{Abp} 1$, a component that can recruit interaction partners of its $\mathrm{SH} 3$ domain to dynamic F-actin structures of the cell cortex (Fenster et al., 2003; Qualmann et al., 2004). Indeed, Cobl and Abp1 colocalized in F-actin-rich lamellipodia of non-neuronal cells and at F-actin-rich sites in the molecular layer of the cerebellum. Furthermore, our subcellular fractionations showed that Cobl and Abp1 both floated with plasma membrane material to which actin was associated.

Interestingly, Ravanelli and Klingensmith (2011) reported that mouse Cobl proteins overexpressed in zebrafish were located at the cell periphery by some unknown mechanism only when $\mathrm{N}$-terminal regions were included, whereas the F-actin binding and nucleating C-terminal WH2 domains (Ahuja et al., 2007) were not sufficient for cortical localization. In line, our coprecipitation studies, coimmunoprecipitation experiments, and in vivo reconstitutions at cellular membranes consistently showed that specifically the N-terminal part of Cobl associates with Abp1. This Abp1 interaction was distinct from interactions of KrRAPpPP-motifs within the Cobl N-terminus with the SH3 domain of syndapin I (Schwintzer et al., 2011), as shown by the successful generation of an Abp1-binding-deficient yet still syndapin-binding mutant. The finding that Cobl was effectively recruited to subcellular membranes presenting the Abp1 SH3 domain clearly demonstrated that the Cobl/Abp1 interaction is strong enough for subcellular recruitment. Finally, coimmunoprecipitations of the endogenous proteins from brain material and the colocalizations of Cobl and Abpl in hippocampal neurons and Purkinje cells demonstrated that Cobl/Abp1 complexes exist in the brain.

Our data strongly suggest that $\mathrm{Cobl}$ and Abp1 work together in promoting neuronal differentiation. This conclusion is highlighted by the fact that Cobl-mediated effects in dendritic branching of hippocampal neurons critically depended on Abp1. They were completely suppressed by Abp1 RNAi. Importantly, deficiency for Abp1 very exactly mirrored the branching phenotype caused by Cobl deficiency in Purkinje cells. These data strongly suggest that, in a physiological context, in Purkinje cells embedded in the cerebellar architecture, dendrite branch formation is critically dependent on Cobl and on its interaction partner Abp1.

In Purkinje cell development, Abp1's role seems to be to ensure proper association of its binding partner with cortical F-actin. Consistently, neither a Cobl mutant lacking the ability to associate with Abp1 nor an Abp1 deletion mutant lacking the F-actin binding abilities of Abp1 were able to rescue the loss-offunction phenotypes of Cobl and Abp1. A role of Abp1 as cortical anchor and $\mathrm{F}$-actin connector for Cobl is well in line with related functions of Abp1 in Arp2/3 complex-mediated actin cytoskeletal processes in spine and axon development in mammals and in bristle development in flies (Pinyol et al., 2007; Haeckel et al., 2008; Dharmalingam et al., 2009; Koch et al., 2012).

Abp1 is strongly targeted to dynamic actin filaments, recruits Cobl, and may thus promote Cobl-mediated actin nucleation at such sites of the cell cortex. As a result, more Abp1 will be recruited and will then either recruit N-WASP and release its autoinhibition (Pinyol et al., 2007) or recruit more Cobl (Fig. 9A). This represents a strong feed-forward mechanism that functionally integrates Arp2/3 complex- and Cobl-mediated actin nucleation at particular sites of the cell cortex and thereby overcomes the tension forces counteracting the formation of protrusive structures from dendrites, which subsequently are established firmly as novel dendritic branches. Consistently, rescue experiments with mutant Cobl and Abp1 showed that Cobl/Abp1 complex formation and F-actin binding of Abp1 are crucial for Cobl and Abpl functions in Purkinje cell arborization. Further in line with the proposed functions of Abp1 (Fig. 9A), previous examinations showed that, in dissociated hippocampal neurons, Coblmediated dendritogenesis is Arp2/3 complex dependent.

Interestingly, in addition to an Abpl-provided feedforward loop, syndapin I interconnects Cobl with the plasma membrane and with Arp $2 / 3$ complex functions. Syndapin I dimers can interact simultaneously with Cobl and the Arp2/3 complex activator N-WASP (Schwintzer et al., 2011), release N-WASP autoinhibition (Dharmalingam et al., 2009), and syndapin I is crucial for Cobl-mediated dendritogenesis and for plasma membrane association of Cobl (Schwintzer et al., 2011). In dissociated hippocampal neurons, syndapin I lossof-function mirrored the dendrite number reductions observed upon Cobl RNAi but failed to show defects on dendritic branching the second Cobl loss-of-function phenotype in hippocampal neurons (Ahuja et al., 2007; Schwintzer et al., 2011). Together, our data strongly suggest that both Abp1 and syndapin I are required for Cobl function but play distinct roles: the F-BAR protein syndapin acts as plasma membrane anchor and curvature sensor (Qualmann et al., 2011) and Abp1 serves as Cobl recruiter to dynamic cortical F-actin (Fig. 9A).

It is thought that proper dendritic arborization of Purkinje cells is a prerequisite for cerebellar motor coordination because only elaborate dendritic trees provide sufficient probability for each parallel fiber running orthogonally through the flat, almost two-dimensional dendritic arbor of the Purkinje cells to make one contact to each Purkinje cell. Interestingly, Abp1 knock-out mice exhibited severe motor coordination deficits in Rotarod and beam walking experiments done before the appearance of any signs of illness or physical abnormalities (Connert et al., 2006). Since neither Cobl nor Abp1 is expressed in considerable amounts in skeletal muscles but both proteins are highly expressed in the brain in adult animals (Kessels et al., 2000; Ahuja et al., 2007), such motor coordination deficits seem to be of neuronal origin and may reflect the Purkinje cell differentiation defects we consistently observed upon deficiency of Cobl and of its interaction partner Abp1.

\section{References}

Ahuja R, Pinyol R, Reichenbach N, Custer L, Klingensmith J, Kessels MM, Qualmann B (2007) Cordon-bleu is an actin nucleation factor and controls neuronal morphology. Cell 131:337-350. CrossRef Medline

Campellone KG, Welch MD (2010) A nucleator arms race: cellular control of actin assembly. Nat Rev Mol Cell Biol 11:237-251. CrossRef Medline

Carroll EA, Gerrelli D, Gasca S, Berg E, Beier DR, Copp AJ, Klingensmith J (2003) Cordon-bleu is a conserved gene involved in neural tube formation. Dev Biol 262:16-31. CrossRef Medline

Chesarone MA, DuPage AG, Goode BL (2010) Unleashing formins to re- 
model the actin and microtubule cytoskeletons. Nat Rev Mol Cell Biol 11:62-74. CrossRef Medline

Conde C, Cáceres A (2009) Microtubule assembly, organization and dynamics in axons and dendrites. Nat Rev Neurosci 10:319-332. CrossRef Medline

Connert S, Wienand S, Thiel C, Krikunova M, Glyvuk N, Tsytsyura Y, Hilfiker-Kleiner D, Bartsch JW, Klingauf J, Wienands J (2006) SH3P7/ mAbp1 deficiency leads to tissue and behavioral abnormalities and impaired vesicle transport. EMBO J 25:1611-1622. CrossRef Medline

Dharmalingam E, Haeckel A, Pinyol R, Schwintzer L, Koch D, Kessels MM, Qualmann B (2009) F-BAR proteins of the syndapin family shape the plasma membrane and are crucial for neuromorphogenesis. J Neurosci 29:13315-13327. CrossRef Medline

Dotti CG, Sullivan CA, Banker GA (1988) The establishment of polarity by hippocampal neurons in culture. J Neurosci 8:1454-1468. Medline

Fenster SD, Kessels MM, Qualmann B, Chung WJ, Nash J, Gundelfinger ED, Garner CC (2003) Interactions between Piccolo and the actin/dynaminbinding protein Abp1 link vesicle endocytosis to presynaptic active zones. J Biol Chem 278:20268-20277. CrossRef Medline

Gasca S, Hill DP, Klingensmith J, Rossant J (1995) Characterization of a gene trap insertion into a novel gene, cordon-bleu, expressed in axial structures of the gastrulating mouse embryo. Dev Genet 17:141-154. CrossRef Medline

Georges PC, Hadzimichalis NM, Sweet ES, Firestein BL (2008) The yinyang of dendrite morphology: unity of actin and microtubules. Mol Neurobiol 38:270-284. CrossRef Medline

Haeckel A, Ahuja R, Gundelfinger ED, Qualmann B, Kessels MM (2008) The actin-binding protein Abpl controls dendritic spine morphology and is important for spine head and synapse formation. J Neurosci 28:1003110044. CrossRef Medline

Kessels MM, Qualmann B (2002) Syndapins integrate N-WASP in receptormediated endocytosis. EMBO J 21:6083-6094. CrossRef Medline

Kessels MM, Engqvist-Goldstein AE, Drubin DG (2000) Association of mouse actin-binding protein 1 (mAbp1/SH3P7), an Src kinase target, with dynamic regions of the cortical actin cytoskeleton in response to Racl activation. Mol Biol Cell 11:393-412. Medline

Kessels MM, Engqvist-Goldstein AE, Drubin DG, Qualmann B (2001) Mammalian Abp1, a signal-responsive F-actin-binding protein, links the actin cytoskeleton to endocytosis via the GTPase dynamin. J Cell Biol 153:351-366. CrossRef Medline

Kessels MM, Schwintzer L, Schlobinski D, Qualmann B (2011) Controlling actin cytoskeletal organization and dynamics during neuronal morphogenesis. Eur J Cell Biol 90:926-933. CrossRef Medline

Koch N, Dharmalingam E, Westermann M, Qualmann B, Thomas U, Kessels MM (2012) Abp1 utilizes the Arp2/3 complex activator Scar/WAVE in bristle development. J Cell Sci 125:3578-3589. CrossRef Medline

Kretzschmar E, Bui M, Rose JK (1996) Membrane association of influenza virus matrix protein does not require specific hydrophobic domains or the viral glycoproteins. Virology 220:37-45. CrossRef Medline

Miyagi Y, Yamashita T, Fukaya M, Sonoda T, Okuno T, Yamada K, Watanabe
M, Nagashima Y, Aoki I, Okuda K, Mishina M, Kawamoto S (2002) Delphilin: a novel PDZ and formin homology domain-containing protein that synaptically colocalizes and interacts with glutamate receptor delta 2 subunit. J Neurosci 22:803-814. Medline

Pinyol R, Haeckel A, Ritter A, Qualmann B, Kessels MM (2007) Regulation of N-WASP and the Arp2/3 complex by Abp1 controls neuronal morphology. PLoS One 2:e400. CrossRef Medline

Pollard TD (2007) Regulation of actin filament assembly by Arp2/3 complex and formins. Annu Rev Biophys Biomol Struct 36:451-477. CrossRef Medline

Poulain FE, Sobel A (2010) The microtubule network and neuronal morphogenesis: dynamic and coordinated orchestration through multiple players. Mol Cell Neurosci 43:15-32. CrossRef Medline

Qualmann B, Kessels MM (2009) New players in actin polymerizationWH2-domain-containing actin nucleators. Trends Cell Biol 19:276-285. CrossRef Medline

Qualmann B, Roos J, DiGregorio PJ, Kelly RB (1999) Syndapin I, a synaptic dynamin-binding protein that associates with the neural Wiskott-Aldrich syndrome protein. Mol Biol Cell 10:501-513. Medline

Qualmann B, Boeckers TM, Jeromin M, Gundelfinger ED, Kessels MM (2004) Linkage of the actin cytoskeleton to the postsynaptic density via direct interactions of Abp1 with the ProSAP/Shank family. J Neurosci 24:2481-2495. CrossRef Medline

Qualmann B, Koch D, Kessels MM (2011) Let's go bananas: revisiting the endocytic BAR code. EMBO J 30:3501-3515. CrossRef Medline

Ravanelli AM, Klingensmith J (2011) The actin nucleator Cordon-bleu is required for development of motile cilia in zebrafish. Dev Biol 350:101111. CrossRef Medline

Schumacher N, Borawski JM, Leberfinger CB, Gessler M, Kerkhoff E (2004) Overlapping expression pattern of the actin organizers Spir-1 and formin-2 in the developing mouse nervous system and the adult brain. Gene Expr Patterns 4:249-255. CrossRef Medline

Schwintzer L, Koch N, Ahuja R, Grimm J, Kessels MM, Qualmann B (2011) The functions of the actin nucleator Cobl in cellular morphogenesis critically depend on syndapin I. EMBO J 30:3147-3159. CrossRef Medline

Soderling SH, Langeberg LK, Soderling JA, Davee SM, Simerly R, Raber J, Scott JD (2003) Loss of WAVE-1 causes sensorimotor retardation and reduced learning and memory in mice. Proc Natl Acad Sci U S A 100: 1723-1728. CrossRef Medline

Sotelo C (2004) Cellular and genetic regulation of the development of the cerebellar system. Prog Neurobiol 72:295-339. CrossRef Medline

Takeuchi T, Ohtsuki G, Yoshida T, Fukaya M, Wainai T, Yamashita M, Yamazaki Y, Mori H, Sakimura K, Kawamoto S, Watanabe M, Hirano T, Mishina M (2008) Enhancement of both long-term depression induction and optokinetic response adaptation in mice lacking delphilin. PLoS One 3:e2297. CrossRef Medline

Yuste R, Bonhoeffer T (2004) Genesis of dendritic spines: insights from ultrastructural and imaging studies. Nat Rev Neurosci 5:24-34. CrossRef Medline 